\title{
CURVATURE TENSORS ON ALMOST HERMITIAN MANIFOLDS
}

\author{
BY \\ FRANCO TRICERRI ${ }^{1}$ AND LIEVEN VANHECKE
}

\begin{abstract}
ABSTRACr. A complete decomposition of the space of curvature tensors over a Hermitian vector space into irreducible factors under the action of the unitary group is given. The dimensions of the factors, the projections, their norms and the quadratic invariants of a curvature tensor are determined. Several applications for almost Hermitian manifolds are given. Conformal invariants are considered and a general Bochner curvature tensor is introduced and shown to be a conformal invariant. Finally curvature tensors on four-dimensional manifolds are studied in detail.
\end{abstract}

1. Introduction. Let $(V, g)$ be an $n$-dimensional real vector space with positive definite inner product $g$ and denote by $\Re(V)$ the subspace of $V^{*} \otimes V^{*} \otimes V^{*} \otimes$ $V^{*}$ consisting of all tensors having the same symmetries as the curvature tensor of a Riemannian manifold, including the first Bianchi identity. In a well-known paper [21] Singer and Thorpe considered $R(V)$ (in particular for $n=4$ ) and gave a geometrical useful description of the splitting of $\mathscr{R}(V)$ under the action of $\theta(n)$ into three components. This was also studied by Nomizu [18] for generalized curvature tensor fields.

A similar decomposition was given in [16], [17] and [22] when $V$ is a $2 n$-dimensional real vector space endowed with a complex structure $J$ compatible with a positive definite inner product $g$ and for the subspace $\mathcal{K}(V)$ of $\mathcal{R}(V)$ consisting of tensors satisfying the Kähler identity. In this case the splitting of $\mathcal{K}(V)$ is treated for the action of $U(n)$.

Of course all these decompositions are, in principle, consequences of general theorems on group representations (see [34]). (For other decompositions see [11], [13].) On the other hand these decompositions do provide insight in some problems of differential geometry. For example, in the splitting of $\mathcal{R}(V)$, one of the projection operators gives the Weyl conformal tensor and in the splitting of $\mathscr{K}(V)$ one obtains in the same way the Bochner tensor. Also the decomposition provides some inequalities for the quadratic invariants and in other cases for some cubic invariants of the curvature tensor. As is well known, these inequalities are very

\footnotetext{
Received by the editors July 3, 1980 and, in revised form, November 4, 1980.

AMS (MOS) subject classifications (1970). Primary 53B20, 53B25, 53B35, 53C15, 53C20, 53C25, $53 \mathrm{C} 55$.

Key words and phrases. Curvature tensors, decomposition into irreducible components, unitary group, quadratic invariants, almost Hermitian manifolds, conformal invariants, Weyl tensor, Bochner tensor, four-dimensional manifolds, submanifolds.

${ }^{1}$ Member of the group G.N.S.A.G.A., C.N.R., Italia.
} 
useful for characterizing some particular manifolds as, for example, Einstein spaces, spaces of constant (holomorphic) sectional curvature, etc.

In this paper we will give the complete decomposition of $\Re(V)$ under the action of $\mathcal{U}(n)$. We start in $\S 2$ with some useful definitions and treat in $\$ 3$ a first partial decomposition by introducing some fundamental linear operators. The operators are involved in some identities for the curvature operator, proved to be useful in studying almost Hermitian manifolds [10], [12]. Then, in \$4, we determine all invariant curvature tensors and in $\$ 5$ we give the complete decomposition.

One of the main results of $\$ 6$ gives the irreducibility of the decomposition. The proof is based on a detailed treatment of the quadratic invariants of the curvature operator. The study of these invariants is also interesting because they are used in several theories on differential geometry such as the theory of the volumes of geodesic spheres and tubes, spectrum theory and some topological and algebraic studies (e.g. the determination of the Euler-Poincaré characteristic, arithmetic genus, Hirzebruch signature, etc.).

In $\$ 7$ we determine explicitly the dimensions of all the factors in the decomposition and in $\S 8$ we write down the projections of a curvature tensor on these factors. This enables us to determine the norms of the projections and so this again provides a method to characterize some particular curvature tensors by using new inequalities. Some applications are given in $\$ \S 10$ and 12 where we consider the Riemannian curvature tensor on a general almost Hermitian manifold. There we consider special classes of manifolds based on several curvature identities and inequalities for the quadratic invariants. Those are introduced in a natural way and are a key to understanding the geometry of almost Hermitian manifolds.

In $\$ 9$ we introduce the notion of a Bochner tensor associated with a curvature tensor and study it in detail in $\$ 10$. Our method to define this tensor is a natural generalization of the Kähler case. Moreover we consider conformal changes of the metric on a Riemanian manifold and the main result is that, just as in the case of the Weyl tensor, the Bochner tensor associated with the Riemann curvature tensor is a conformal invariant tensor. At the same time we discover also other conformal invariant tensors.

In $\$ 13$ we illustrate the decomposition by considering the Calabi-Eckmann manifolds and a Hopf manifold. It would be interesting to illustrate the decomposition with a lot of other examples by constructing manifolds such that the Riemann curvature tensor has one or more vanishing projections on the factors of the decomposition. We refer to [7], [11] and other papers where many examples of almost Hermitian manifolds are given but we mention that there are still some open problems.

Additional motivation for the study of our generalized Bochner tensor is given in $\S \S 14$ and 15. There we treat in detail the decomposition for the case of a four-dimensional vector space. Four-dimensional geometry has a special feature because of the *-operator and is intensively studied at present in relation to Yang-Mills theory [1], [8]. In particular we study the self-dual and anti-self-dual part of the Weyl conformal tensor and we show, for example, that on a Hermitian 
manifold the Bochner tensor is just the anti-self-dual part of it. Further we compute the Euler-Poincare characteristic, the Hirzebruch signature and the arithmetic genus for compact orientable Einstein spaces and derive several useful inequalities between them.

Finally in $\S 15$ we consider submanifolds of almost Hermitian manifolds and give a theorem concerning the relation between the Bochner tensor of the ambient space and the Weyl tensor of a totally real submanifold. This again illustrates how both tensors have an important place in conformal geometry.

The authors wish to thank A. Gray for his interest in our work and for several useful discussions.

2. Preliminaries. Let $V$ be an $n$-dimensional real vector space with positive definite inner product $g$. A tensor $R$ of type $(1,3)$ over $V$ is a bilinear mapping

$$
R: V \times V \rightarrow \operatorname{Hom}(V, V):(x, y) \mapsto R(x, y) \text {. }
$$

$R$ is called a curvature tensor over $V$ if it has the following properties for all $x, y, z, w \in V$ :

(i) $R(x, y)=-R(y, x)$;

(ii) $R(x, y)$ is a skew-symmetric endomorphism of $V$, i.e.

$$
R(x, y, z, w)+R(x, y, w, z)=0 \quad \text { where } R(x, y, z, w)=g(R(x, y) z, w) ;
$$

(iii) $\subseteq R(x, y) z=0$ where $\subseteq$ denotes the cyclic sum over $x, y$ and $z$. This is the first Bianchi identity.

The Ricci tensor $\rho(R)$ of type $(0,2)$ associated with $R$ is a symmetric bilinear function on $V \times V$ defined by

$$
\rho(R)(x, y)=\operatorname{trace}(z \in V \mapsto R(x, z) y \in V) .
$$

Then, the Ricci tensor $Q=Q(R)$ of type $(1,1)$ is given by $\rho(R)(x, y)=g(Q x, y)$ and the trace of $Q$ is called the scalar curvature $\tau=\tau(R)$ of $R$.

Next, let $V$ be a $2 n$-dimensional real vector space with a complex structure $J$ and a Hermitian product $g$, i.e.

$$
J^{2}=-I, \quad g(J x, J y)=g(x, y)
$$

for all $x, y \in V$, and where $I$ denotes the identity transformation of $V$.

$\rho$ is a $\mathcal{U}(n)$-concomitant [3]. Further we define a second $\mathscr{U}(n)$-concomitant $\rho^{*}$ which, together with $\rho$ and $J$, suffices to write down all $\mathcal{U}(n)$-concomitants of $\mathcal{R}(V)$ in $\Lambda^{2}(V)$ following the methods of [15].

The Ricci *-tensor $\rho^{*}(R)$ of type $(0,2)$, resp. $Q^{*}=Q^{*}(R)$ of type $(1,1)$, associated with a curvature tensor $R$ is defined by

$$
\begin{aligned}
\rho^{*}(R)(x, y) & =g\left(Q^{*} x, y\right)=\operatorname{trace}(z \in V \mapsto R(J z, x) J y \in V) \\
& =\sum_{i=1}^{2 n} R\left(x, e_{i}, J y, J e_{i}\right)
\end{aligned}
$$

where $\left\{e_{i}, i=1, \ldots, 2 n\right\}$ is an arbitrary orthonormal basis of $V$. Then, the trace of $Q^{*}$ is called the $*$-scalar curvature $\tau^{*}=\tau^{*}(R)$ of $R$. In general $\rho^{*}(R)$ is neither 
symmetric nor antisymmetric but we always have

$$
\rho^{*}(R)(x, y)=\rho^{*}(R)(J y, J x)
$$

for all $x, y \in V$.

We note that for some classes of almost Hermitian manifolds it is possible to express the first Chern class with the help of $\rho(R)$ and $\rho^{*}(R)$. This is done in [9] for the class of nearly Kähler manifolds although the $\rho^{*}(R)$ is slightly different from the one just defined.

Let $R(V)$ denote the vector space of all curvature tensors over $V$. This space has a natural inner product induced from that on $V$ :

$$
\langle R, \tilde{R}\rangle=\sum_{i, j, k=1}^{2 n} g\left(R\left(e_{i}, e_{j}\right) e_{k}, \tilde{R}\left(e_{i}, e_{j}\right) e_{k}\right)
$$

where $R, \tilde{R} \in \mathcal{R}(V)$ and $\left\{e_{i}\right\}$ is an arbitrary orthonormal basis of $V$. Further, let $\alpha$ be the standard representation of the unitary group $\mathcal{U}(n)$ in $V$. Then there is a natural induced representation $\tilde{\alpha}$ of $\mathcal{U}(n)$ in $\mathcal{R}(V)$ given by

$$
\tilde{\alpha}(a)(R)(x, y, z, w)=R\left(\alpha\left(a^{-1}\right) x, \alpha\left(a^{-1}\right) y, \alpha\left(a^{-1}\right) z, \alpha\left(a^{-1}\right) w\right)
$$

for all $x, y, z, w \in V, R \in \mathcal{R}(V)$ and $a \in \mathcal{Q}(n)$.

We have

$$
\langle\tilde{\alpha}(a) R, \tilde{\alpha}(a) \tilde{R}\rangle=\langle R, \tilde{R}\rangle, \quad a \in \mathcal{U}(n), \quad R, \tilde{R} \in \mathcal{R}(V) .
$$

This implies that the orthogonal complement of an invariant subspace of $R(V)$ is also invariant. Further it follows from this that the standard representation of $\mathscr{U}(n)$ in $\mathscr{R}(V)$ is completely reducible.

Finally we note that $\operatorname{dim} R(V)=1$ when $n=1$. In that case $\Re(V)$ is irreducible. Hence, from now on we only consider the case $n \geqslant 2$.

3. Partial decomposition. Our aim is to give a complete decomposition of $\mathscr{R}(V)$ into orthogonal irreducible factors. Before doing this we introduce three particular subspaces $\mathcal{R}_{i}(V), i=1,2,3$, of $\mathcal{R}(V)$, following [10].

DeFINITION 3.1.

$$
\begin{gathered}
\Re_{1}(V)=\{R \in \mathcal{R}(V) \mid R(x, y, z, w)=R(x, y, J z, J w)\} ; \\
\Re_{2}(V)=\{R \in R(V) \mid R(x, y, z, w)=R(J x, J y, z, w) \\
\quad+R(J x, y, J z, w)+R(J x, y, z, J w)\} ; \\
\Re_{3}(V)=\{R \in R(V) \mid R(x, y, z, w)=R(J x, J y, J z, J w)\} .
\end{gathered}
$$

These subspaces are invariant under the action of $\mathcal{Q}(n)$. Further we have (see, for example, [10], [12]): $\Re_{1}(V) \subset \Re_{2}(V) \subset \Re_{3}(V)$.

Next we put:

$\Re_{1}^{\perp}(V)=$ orthogonal complement of $\Re_{1}(V)$ in $\Re_{2}(V)$;

$\Re_{2}^{\perp}(V)=$ orthogonal complement of $\Re_{2}(V)$ in $\Re_{3}(V)$;

$\Re_{3}^{\perp}(V)=$ orthogonal complement of $\Re_{3}(V)$ in $\Re(V)$.

Hence we have already the following decomposition of $\mathcal{R}(V)$ into orthogonal invariant subspaces. 
THEOREM 3.2. We have

$$
\Re(V)=\Re_{1}(V) \oplus \Re_{1}^{\perp}(V) \oplus \Re_{2}^{\perp}(V) \oplus \Re_{3}^{\perp}(V) .
$$

In order to describe and to study these subspaces more in detail we put $\Re_{4}(V)=\Re(V)$ and introduce three linear operators $L_{i}, i=1,2,3$. As mentioned in the Introduction, these operators are closely related to some identities for the curvature operator, proven to be useful in the study of almost Hermitian manifolds [10], [12].

Definition 3.3. For all $x, y, z, w \in V$ we put

(a)

$$
\left(L_{1} R\right)(x, y, z, w)=\frac{1}{2}\{R(J x, J y, z, w)+R(y, J z, J x, w)+R(J z, x, J y, w)\}
$$

for all $R \in \mathscr{R}_{2}(V)$;

(b)

$$
\begin{aligned}
\left(L_{2} R\right)(x, y, z, w)=\frac{1}{2}\{ & R(x, y, z, w)+R(J x, J y, z, w) \\
+ & R(J x, y, J z, w)+R(J x, y, z, J w)\}
\end{aligned}
$$

for all $R \in \mathscr{R}_{3}(V)$;

(c)

$$
\left(L_{3} R\right)(x, y, z, w)=R(J x, J y, J z, J w) \text { for all } R \in R(V) .
$$

It follows from this that

$$
\left(L_{1} R\right)(x, y, z, w)-\left(L_{1} R\right)(x, y, J z, J w)=-R(x, y, z, w)+R(x, y, J z, J w)
$$

and hence we have

LEMMA 3.4. $L_{1} R=R$ if and only if $R \in \Re_{1}(V)$.

Further we obtain

LEMMA 3.5. The restriction of $L_{i}, i=1,2,3$, to $\Re_{i+1}$ is an involutive isometry. Moreover, $L_{i}$ commutes with the action of $\mathcal{U}(n)$, i.e. $L_{i}$ is a U(n)-concomitant [3].

This lemma implies that $L_{i}$ has two eigenvalues, +1 and -1 , and that the corresponding eigenspaces are orthogonal invariant subspaces of $\Re_{i+1}(V)$.

Since $\frac{1}{2}\left(R+L_{i} R\right)$, respectively $\frac{1}{2}\left(R-L_{i} R\right)$, is an eigenvector of $L_{i}$ for $R \in$ $\Re_{i+1}(V)$, corresponding to the eigenvalue +1 , respectively -1 , each $R \in \Re_{i+1}(V)$ can be decomposed uniquely and orthogonally as follows:

$$
R=\frac{1}{2}\left(R+L_{i} R\right)+\frac{1}{2}\left(R-L_{i} R\right) .
$$

The components of the decomposition (3.1) are now characterized as follows:

TheOREM 3.6. We have for $i=1,2,3: \Re_{i}(V)=(+1)$-eigenspace of $L_{i} ; R_{i}{ }^{\perp}(V)=$ (-1)-eigenspace of $L_{i}$.

Proof. This follows easily from Definition 3.3, Lemma 3.4 and the remarks above.

Using Lemma 3.4 and formula (3.3) it is not difficult to write down the projections of a curvature tensor $R$ on the components of the decomposition (3.1). We will treat this in detail in $\$ 9$. 
Further we note that (3.1) is not irreducible. Indeed the following theorem is well known.

THEOREM 3.7 [16], [17], [22]. We have $\Re_{1}(V)=\mathscr{W}_{1} \oplus \mathscr{W}_{2} \oplus \mathscr{W}_{3}$, where:

$\mho_{1}=\left\{R \in \mathscr{R}_{1}(V)\right.$ with constant holomorphic sectional curvature $\}$;

$\mathcal{W r}_{3}=\left\{R \in \mathcal{R}_{1}(V) \mid \rho(R)=0\right\}$;

$\mho_{2}=$ orthogonal complement of $\mho_{1} \oplus \mho_{3}$.

Note that $\mathscr{W}_{2} \oplus \mathscr{W}_{3}=\left\{R \in \mathscr{R}_{1}\right.$ with $\left.\tau(R)=0\right\}$.

Finally, we give some lemmas which we need in what follows.

LEMMA 3.8. $R \in \Re_{2}^{\perp}(V)$ if and only if for all $x, y, z, w \in V$ :

$$
R(J x, y, z, w)=R(x, J y, z, w)=R(x, y, J z, w)=R(x, y, z, J w) .
$$

Proof. We always have

$$
\left(L_{2} R\right)(J x, y, z, w)-\left(L_{2} R\right)(x, y, z, J w)=R(J x, y, z, w)-R(x, y, z, J w) .
$$

Since $R \in \mathscr{R}_{2}^{\perp}(V)$ we have $L_{2} R=-R$ and, hence, $R(J x, y, z, w)=R(x, y, z, J w)$. The other equalities follow by using the properties of the curvature tensor.

The converse follows at once from the definition.

By explicit calculations we obtain the following series of lemmas.

LEMmA 3.9. We have, for all $R \in R(V)$ and all $x, y \in V$ :

(i) $\rho\left(L_{3} R\right)(x, y)=\rho(R)(J x, J y)$;

(ii) $\rho^{*}\left(L_{3} R\right)(x, y)=\rho^{*}(R)(J x, J y)$.

LEMMa 3.10. For all $R \in \Re_{3}(V)$ we have:

(i) $\rho\left(L_{2} R\right)=\rho(R)$;

(ii) $\rho^{*}\left(L_{2} R\right)=\rho^{*}(R)$.

LEMMA 3.11. We have for all $R \in \Re_{2}(V)$ :

(i) $2 \rho\left(L_{1} R\right)=3 \rho^{*}(R)-\rho(R)$;

(ii) $2 \rho^{*}\left(L_{1} R\right)=\rho(R)+\rho^{*}(R)$.

Using these lemmas and (2.1) we obtain

LEMMA 3.12. For $R \in \mathcal{R}_{3}(V), \rho(R)$ and $\rho^{*}(R)$ are symmetric and

$$
\rho(R)(J x, J y)=\rho(R)(x, y), \quad \rho^{*}(R)(J x, J y)=\rho^{*}(R)(x, y)
$$

for all $x, y \in V$.

LEMMA 3.13. For $R \in \Re_{3}^{\perp}(V), \rho(R)$ is symmetric and $\rho^{*}(R)$ is antisymmetric. Further

$$
\rho(R)(J x, J y)=-\rho(R)(x, y), \quad \rho^{*}(R)(J x, J y)=-\rho^{*}(R)(x, y)
$$

for all $x, y \in V$.

LEMMA 3.14. $\rho(R)=\rho^{*}(R)=0$ for $R \in \mathcal{R}_{2}^{\perp}(V)$.

LEMMA 3.15. $\rho(R)=\rho^{*}(R)$ for $R \in \Re_{1}(V)$ and $\rho(R)+3 \rho^{*}(R)=0$ for $R \in$ $\Re_{1}^{\perp}(V)$. 
4. Invariant curvature tensors. A curvature tensor $R$ is invariant under the action of $\mathcal{U}(n)$ if and only if $\tilde{\alpha}(a)(R)=R$ for all $a \in \mathcal{Q}(n)$. Such a curvature tensor determines a 1-dimensional invariant and irreducible subspace of $\Re(V)$. We now determine these tensors.

THEOREM 4.1. The set of invariant curvature tensors of $\Re(V)$ forms a 2-dimensional subspace of $R(V)$ generated by the tensors $\pi_{1}$ and $\pi_{2}$ defined as follows:

$$
\begin{aligned}
& \pi_{1}(x, y) z=g(x, z) y-g(y, z) x, \\
& \pi_{2}(x, y) z=2 g(J x, y) J z+g(J x, z) J y-g(J y, z) J x
\end{aligned}
$$

for all $x, y, z \in V$.

Proof. A result of Iwahori [15] implies that the invariant tensors of type $(0,4)$ form a 12-dimensional vector space with basis $\left\{F_{\alpha_{1} \alpha_{2}}^{o}\right\}$ for $n \geqslant 2$ where

$$
F_{\alpha_{1} \alpha_{2}}^{\sigma}\left(x_{1}, x_{2}, x_{3}, x_{4}\right)=\Omega_{\alpha_{1}}\left(x_{\sigma(1)}, x_{\sigma(2)}\right) \Omega_{\alpha_{2}}\left(x_{\sigma(3)}, x_{\sigma(4)}\right),
$$

$\sigma$ being the identity or one of the following permutations: $(1,2,3,4) \rightarrow(1,3,2,4)$, $(1,2,3,4) \rightarrow(1,4,3,2)$. Further, $\alpha_{1}, \alpha_{2}=0,1$ and $\Omega_{0}(x, y)=g(x, y), \Omega_{1}(x, y)=$ $g(x, J y)$. The required result now follows by direct computation and by using the symmetries of the curvature tensor.

For these invariant curvature tensors we now give some useful properties.

LEMMA 4.2. (i) $L_{3} \pi_{1}=\pi_{1}, L_{3} \pi_{2}=\pi_{2}$;

(ii) $L_{2} \pi_{1}=\pi_{1}, L_{2} \pi_{2}=\pi_{2}$;

(iii) $2 L_{1} \pi_{1}=\pi_{2}-\pi_{1}$.

(i) and (ii) imply that $\pi_{1}$ and $\pi_{2}$ are elements of $\Re_{2}(V)$ and since $L_{1}$ is involutive, (iii) gives

$$
L_{1} \pi_{2}=2 \pi_{1}+L_{1} \pi_{1}
$$

Hence:

LEMMA 4.3. We have

$$
\pi_{1}+\pi_{2} \in \Re_{1}(V) \text { and } 3 \pi_{1}-\pi_{2} \in \Re_{1}^{\perp}(V) .
$$

It is known that a tensor $R \in \mathscr{R}_{1}(V)$ has constant holomorphic sectional curvature $4 \lambda$ if and only if $R=\lambda\left(\pi_{1}+\pi_{2}\right)$. Hence we obtain

THEOREM 4.4. $\mathscr{W} \int_{1}=\mathcal{L}\left(\pi_{1}+\pi_{2}\right)$, where $\mathcal{L}\left(\pi_{1}+\pi_{2}\right)$ denotes the vector space spanned by $\pi_{1}+\pi_{2}$.

Further, it is easy to show

LEMMA 4.5. (i) $\left\langle R, \pi_{1}\right\rangle=2 \tau(R)$; (ii) $\left\langle R, \pi_{2}\right\rangle=6 \tau^{*}(R)$ for all $R \in \Re(V)$.

DEFINITION 4.6. $\mathscr{W}_{4}=\mathcal{L}\left(3 \pi_{1}-\pi_{2}\right)$ is the vector space generated by the curvature tensor $3 \pi_{1}-\pi_{2}$.

Then we obtain from Lemma 4.5

THEOREM 4.7. The orthogonal complement of $\mathscr{W}_{1} \oplus \mathcal{W}_{4}$ in $R(V)$ consists of curvature tensors $R \in \Re(V)$ such that $\tau(R)=\tau^{*}(R)=0$. 
5. The complete decomposition. Before giving the fundamental theorem we shall define all the components of the decomposition.

DEFINITION 5.1. We put

थิ $\mathcal{L}_{1}=\mathcal{L}\left(\pi_{1}+\pi_{2}\right)$,

$\mathcal{W}_{3}=\left\{R \in \mathcal{R}_{1}(V) \mid \rho(R)=0\right\}$,

$\mathcal{W}_{2}=$ orthogonal complement of $\mathscr{W}_{1} \oplus \mathcal{W}_{3}$ in $\mathcal{R}_{1}(V)$,

$\mathscr{W}_{4}=\mathcal{L}\left(3 \pi_{1}-\pi_{2}\right) \subset \Re_{1}^{\perp}(V)$,

$\mathscr{w}_{6}=\left\{R \in \Re_{1}^{\perp}(V) \mid \rho(R)=0\right\}$,

$\mathcal{W S}_{5}=$ orthogonal complement of $\mho_{4} \oplus \mathcal{W}_{6}$ in $\mathcal{R}_{1}^{\perp}(V)$,

$\mathrm{W}_{7}=\Re_{2}^{\perp}(V)$,

$\mathscr{W}_{10}=\left\{R \in \mathscr{R}_{3}^{\perp}(V) \mid \rho(R)=\rho^{*}(R)=0\right\}$,

$\mathcal{W}_{8} \oplus \mathcal{W}_{9}=$ orthogonal complement of $\mathscr{W}_{10}$ in $\Re_{3}^{\perp}(V)$,

$\mathcal{W}_{8}=\left\{R \in \mathcal{W}_{8} \oplus \mathcal{W}_{9} \mid \rho^{*}(R)=0\right\}$,

$\mathcal{W}_{9}=$ orthogonal complement of $W_{8}$ in $\mho_{8} \oplus \mho_{9}$.

We now obtain at once

THEOREM 5.2.

$$
\Re(V)=\mho_{1} \oplus \cdots \oplus W_{10}
$$

where the $\mathcal{W}_{i}$ are orthogonal invariant subspaces.

Next we recall that an invariant subspace is irreducible when it does not contain a nontrivial invariant subspace. Now we state the fundamental theorem of this paper.

THEOREM 5.3. (i) The decomposition (5.1) is irreducible for $n>4$.

(ii) For $n=3, \mathscr{Q} \int_{6}=\{0\}$ and the other factors in (5.1) are irreducible.

(iii) For $n=2$, $\mathcal{W}_{5}=\mathcal{W}_{6}=\mathcal{W}_{10}=\{0\}$ and the other factors in (5.1) are irreducible.

We postpone the proof to $\S 6$. We shall need algebraic characterizations of $\mho_{2}$, $\mho_{5}, \mho_{8}$ and $\mho_{9}$ and so we do this first.

Definition 5.4. Let $V^{*}$ denote the dual space of $V$ and let $S \in \otimes^{2} V^{*}=V^{*} \otimes$ $V^{*}$. Define $\varphi(S)$ and $\psi(S)$ by

$$
\begin{aligned}
\varphi(S)(x, y, z, w)= & g(x, z) S(y, w)+g(y, w) S(x, z) \\
& -g(x, w) S(y, z)-g(y, z) S(x, w) \\
\psi(S)(x, y, z, w)= & 2 g(x, J y) S(z, J w)+2 g(z, J w) S(x, J y) \\
& +g(x, J z) S(y, J w)+g(y, J w) S(x, J z) \\
& -g(x, J w) S(y, J z)-g(y, J z) S(x, J w) .
\end{aligned}
$$

$\varphi$ and $\psi$ are $\mathcal{Q}(n)$-concomitants of $\otimes^{2} V^{*}$ in $\otimes^{4} V^{*}$ since they commute with the action of $\mathscr{U}(n)$. In general the values do not belong to $\mathcal{R}(V)$ but we have the following result.

THEOREM 5.5. (i) $\varphi(S) \in \mathcal{R}(V)$ if and only if $S$ is symmetric.

(ii) $\psi(S) \in \Re(V)$ if and only if $S(x, J y)+S(y, J x)=0$ for all $x, y \in V$.

Proof. Let $S$ be symmetric. Then we see at once that $\varphi(S) \in R(V)$. To prove the converse we choose orthogonal unit vectors $x, y, w$. This is possible because 
$\operatorname{dim} V \geqslant 4$. Since $\varphi(S) \in \mathcal{R}(V)$ we have $\varphi(S)(x, y, x, w)=\varphi(S)(x, w, x, y)$ and this implies that $S$ is symmetric.

Next we suppose $\psi(S) \in \mathcal{R}(V)$. Hence $\psi(S)(x, y, z, w)=-\psi(S)(y, x, z, w)$ and this is equivalent to $g(z, J w)\{S(x, J y)+S(y, J x)\}=0$ for all $x, y, z, w \in V$. So the second factor must be zero. The converse is obtained by a simple verification.

DeFINITION 5.6. For all $x, y \in V$ we put

$$
\begin{aligned}
& \mathscr{V}_{1}=\left\{S \in \otimes^{2} V^{*} \mid S(x, y)=S(y, x), S(J x, J y)=S(x, y), \text { trace } S=0\right\}, \\
& \mathcal{V}_{2}=\left\{S \in \otimes^{2} V^{*} \mid S(x, y)=S(y, x), S(J x, J y)=-S(x, y)\right\}, \\
& \mathscr{V}_{3}=\left\{S \in \otimes^{2} V^{*} \mid S(x, y)=-S(y, x), S(J x, J y)=-S(x, y)\right\} .
\end{aligned}
$$

Then we have

THEOREM 5.7. (i) $\mho_{2}=(\varphi+\psi)\left(\mathscr{V}_{1}\right)$;

(ii) $\mathscr{W}_{5}=(3 \varphi-\psi)\left(\mathscr{V}_{1}\right)$;

(iii) $\mathscr{w}_{8}=\varphi\left(\mathscr{T}_{2}\right)$;

(iv) $\mho_{9}=\psi\left(\mathscr{V}_{3}\right)$.

For the proof of this theorem we shall need a series of lemmas which may be checked easily.

LEMMA 5.8. For all symmetric $S \in \otimes^{2} V^{*}$ we have

(i) $\rho(\varphi(S))=2(n-1) S+\operatorname{tr} S g$;

(ii) $\rho^{*}(\varphi(S))=S+S^{\prime}$ where $S^{\prime}(x, y)=S(J x, J y)$ for all $x, y \in V$;

(iii) $\langle R, \varphi(S)\rangle=4\langle\rho(R), S\rangle$.

Lemma 5.9. Suppose $S \in \otimes^{2} V^{*}$ satisfies $S(x, J y)+S(y, J x)=0$ for all $x, y \in$ $V$. Then we have

(i) $\rho(\psi(S))=3(S+\stackrel{\circ}{S})$;

(ii) $\rho^{*}(\psi(S))=2(n+1) S+\operatorname{tr} S g$;

(iii) $\langle R, \psi(S)\rangle=8\left\langle\rho^{*}(R), S\right\rangle+4\left\langle\rho^{*}(R), \dot{S}\right\rangle$

where $\dot{S}$ is defined by $\dot{S}(x, y)=S(y, x)$ for all $x, y \in V$.

LEMMA 5.10. Let $S \in \mathfrak{V}_{1}$. Then

(i) $L_{3} \varphi(S)=\varphi(S), L_{3} \psi(S)=\psi(S)$;

(ii) $L_{2} \varphi(S)=\varphi(S), L_{2} \psi(S)=\psi(S)$;

(iii) $2 L_{1} \varphi(S)=\psi(S)-\varphi(S)$ and $L_{1} \psi(S)=2 \varphi(S)+L_{1} \varphi(S)$.

Lemma 5.11. Let $S \in \mathfrak{T}_{2}$. Then $L_{3} \varphi(S)=-\varphi(S)$.

Lemma 5.12. Let $S \in \mathcal{V}_{3}$. Then $L_{3} \psi(S)=-\psi(S)$.

Proof OF Theorem 5.7. It follows from Theorem 3.6 and Lemma 5.10 that

$$
(\varphi+\psi)\left(\mathscr{V}_{1}\right) \subset \Re_{1}(V)=\mho_{1} \oplus \mathscr{W}_{2} \oplus \mho_{3}
$$

Moreover, Lemmas 5.8 and 5.9 imply $\tau(R)=\tau^{*}(R)=0$ for all $R \in(\varphi+\psi)\left(\mathscr{V}_{1}\right)$. Hence, using Theorem 4.7, we have $(\varphi+\psi)\left(\mathcal{V}_{1}\right) \subset \mho_{2} \oplus \mho_{3}$. Further, note that when $R \in \mathcal{W}_{2} \oplus \mathscr{W}_{3}$ we have $\rho(R)=\rho^{*}(R)$ (Lemma 3.15) and $\rho(R) \in \mathcal{V}_{1}$ (Lemma 3.12 and Theorem 4.7). Hence, using Lemmas 5.8 and 5.9 we obtain for $R \in \mathcal{W}_{2} \oplus \mathcal{W}_{3}$ and $S \in V_{1}$

$$
\langle R,(\varphi+\psi)(S)\rangle=0 \text { if and only if } R \in \mathscr{\mho}_{3} .
$$

So, we may conclude that $(\varphi+\psi)\left(\mathscr{V}_{1}\right)=\mathscr{W}_{2}$. 
$\mathscr{W}_{5}=(3 \varphi-\psi)\left(\mathscr{V}_{1}\right)$ can be proved in a similar way.

Finally we prove (iii) and (iv). First we note that $\varphi\left(\mathscr{V}_{2}\right) \subset \Re_{3}^{\perp}(V)$ and $\psi\left(\mathscr{V}_{3}\right) \in$ $\Re_{3}^{\perp}(V)$ follow from Lemmas 5.11, 5.12 and Theorem 3.6. Next, Lemmas 5.8 and 5.9 imply that $\varphi\left(\mathscr{V}_{2}\right)$ and $\psi\left(\mathscr{V}_{3}\right)$ are orthogonal. Also for $R \in \Re_{3}^{\perp}(V)$ and $S \in$ $\varphi\left(\mathscr{V}_{2}\right) \oplus \psi\left(\mathscr{V}_{3}\right)$ we have

$$
\rho(R)=\rho^{*}(R)=0 \quad \text { if and only if }\langle R, S\rangle=0
$$

since $\rho(R) \in \mathscr{V}_{2}$ and $\rho^{*}(R) \in \mathcal{V}_{3}$ (Lemma 3.13). Finally $\rho^{*}(S)=0$ if and only if $S \in \varphi\left(\mathscr{V}_{2}\right)$. This proves the required result.

Theorem 5.7 and Lemma 5.9 also imply

THEOREM 5.13. $\mathscr{W}_{9}=\left\{R \in \mathcal{V}_{8} \oplus \mathscr{\partial} \int_{9} \mid \rho(R)=0\right\}$.

We conclude this section with some remarks.

(A) It is immediately seen that $2 \pi_{1}=\varphi(g)$ and $2 \pi_{2}=\psi(g)$ and so Lemmas 4.2 and 4.3 are special cases of Lemmas 5.8-5.10. In addition we obtain from Lemmas 5.8 and 5.9

THEOREM 5.14. We have

(i) $\rho\left(\pi_{1}\right)=(2 n-1) g, \rho\left(\pi_{2}\right)=3 g$;

(ii) $\rho^{*}\left(\pi_{1}\right)=g, \rho^{*}\left(\pi_{2}\right)=(2 n+1) g$;

(iii) $\tau\left(\pi_{1}\right)=2 n(2 n-1), \tau\left(\pi_{2}\right)=6 n$;

(iv) $\tau^{*}\left(\pi_{1}\right)=2 n, \tau^{*}\left(\pi_{2}\right)=2 n(2 n+1)$.

(B) Using the same lemmas it is easily shown that the restrictions of $\varphi$ and $\psi$ to $\mho_{1}$ are linearly independent when $n>2$. For $n=2$ we have $\|3 \varphi(S)-\psi(S)\|=0$ for all $S \in \mathfrak{V}_{1}$. So $3 \varphi(S)=\psi(S)$ or $\mathscr{W}_{5}=\{0\}$.

(C) It is clear that the decomposition (5.1) is not unique.

(D) The results written down in Table I follow from the results above or are easily derived.

\begin{tabular}{|c|c|c|c|c|c|c|c|}
\hline & $\tau(R)$ & $\tau^{*}(R)$ & $\rho(R)$ & $\rho^{*}(R)$ & $L_{3}(R)$ & $L_{2}(R)$ & $L_{1}(R)$ \\
\hline$w_{1}$ & $4 n(n+1) \lambda$ & $4 n(n+1) \lambda$ & $2(n+1) g$ & $2(n+1) g$ & $R$ & $R$ & $R$ \\
\hline$w_{2}$ & 0 & 0 & $2(n+2) S$ & $2(n+2) S$ & $R$ & $R$ & $R$ \\
\hline$w_{3}$ & 0 & 0 & 0 & 0 & $R$ & $R$ & $\bar{R}$ \\
\hline ขึ & $12 n(n-1) \lambda$ & $-4 n(n-1) \lambda$ & $6(n-1) g$ & $-2(n-1) g$ & $R$ & $R$ & $-R$ \\
\hline$w_{5}$ & 0 & 0 & $6(n-2) S$ & $-2(n-2) S$ & $R$ & $R$ & $-R$ \\
\hline$w_{6}$ & 0 & 0 & 0 & 0 & $R$ & $R$ & $-R$ \\
\hline$w_{7}$ & 0 & 0 & 0 & 0 & $R$ & $-R$ & \\
\hline $\mathrm{US}_{8}$ & 0 & 0 & $2(n-1) S$ & 0 & $-R$ & & \\
\hline$w_{9}$ & 0 & 0 & 0 & $2(n+1) S$ & $-R$ & & \\
\hline$w_{10}$ & 0 & 0 & 0 & 0 & $-R$ & & \\
\hline
\end{tabular}

TABLE I 
6. Quadratic invariants and the irreducibility of the decomposition. To prove the irreducibility of the decomposition (5.1) we will use the following characterization: An invariant subspace of $\otimes^{r} V^{*}$ is irreducible for the action of $\mathcal{U}(n)$ (or more generally, for the action of a subgroup $\mathcal{G}$ of $\mathcal{O}(2 n)$ ) if and only if the space of its quadratic invariants is 1-dimensional (see for example [2], [11]).

We note (see [2]) that all the quadratic invariants of a subspace of $\otimes^{r} V^{*}$ are restrictions of quadratic invariants of $\otimes^{r} V^{*}$. These last ones may be written as follows:

$$
P(T)=\sum T\left(e_{i_{1}}, \ldots, e_{i_{r}}\right) T\left(e_{j_{1}}, \ldots, e_{j_{r}}\right) p\left(e_{i_{1}}, \ldots, e_{i_{r}}, e_{j_{1}}, \ldots, e_{j_{r}}\right)
$$

where $T \in \otimes^{r} V^{*}$ and $p \in \otimes^{2 r} V^{*}$ is invariant, i.e.

$$
\tilde{\alpha}(a)(p)\left(x_{1}, \ldots, x_{2 r}\right)=p\left(\tilde{\alpha}\left(a^{-1}\right) x_{1}, \ldots, \tilde{\alpha}\left(a^{-1}\right) x_{2 r}\right)=p\left(x_{1}, \ldots, x_{2 r}\right)
$$

for all $x_{i} \in V$ and $a \in \mathcal{Q}(n)$.

Hence the problem of determining the quadratic invariants is equivalent with the problem of determining the invariant tensors of $\otimes^{2 r} V^{*}$. We will again use the results of [15].

First we have

LEMMA 6.1. The three subspaces $\mathcal{T}_{i}, i=1,2,3$, of $\otimes^{2} V^{*}$ are irreducible.

Proof. Following (6.1), all the quadratic invariants of $\otimes^{2} V^{*}$ are given by

$$
P(S)=\sum S\left(e_{i}, e_{j}\right) S\left(e_{h}, e_{k}\right) p\left(e_{i}, e_{j}, e_{h}, e_{k}\right)
$$

where $S \in \otimes^{2} V^{*}$ and $p$ is an invariant of $\otimes^{4} V^{*}$. Those are linear combinations of the $F_{\alpha_{1} \alpha_{2}}^{\sigma}$ in (4.1). It is easy to see that the restrictions of $P(S)$ to $\mathscr{V}_{1}, \mathscr{V}_{2}, \mathscr{V}_{3}$ are scalar multiples of $\|S\|^{2}$. Moreover we have

$$
\operatorname{dim} \mathscr{V}_{1}=n^{2}-1, \quad \operatorname{dim} \mathscr{V}_{2}=n(n+1), \quad \operatorname{dim} \mathscr{V}_{3}=n(n-1)
$$

Since $n \geqslant 2$, these dimensions are never zero. Hence the required result follows.

Next we note that when $\xi$ is a $\mathcal{G}$-concomitant between two spaces, $\mathcal{G}$ acting on these spaces, then the image for $\xi$ of an invariant subspace is also invariant. Further, the image is irreducible when the first space is irreducible. In this case the restriction of $\xi$ is an isomorphism. (See, for example, [3].)

This remark, Remark (A) of $\S 5$, Theorem 5.7 and Lemma 6.1 imply

THEOREM 6.2. (i) $\mho_{2}, W_{8}$ and $W_{9}$ are irreducible.

(ii) $\mho_{5}=\{0\}$ for $n=2$ and irreducible for $n>2$.

Before proving the irreducibility of the other factors in (5.1), we determine the quadratic invariants.

THEOREM 6.3. The vector space of all quadratic invariants of $R \in \mathcal{R}(V)$ is spanned by the following 12 invariants:

$$
o_{1}(R)=\|R\|^{2}, \quad o_{2}(R)=\|\rho(R)\|^{2}, \quad o_{3}(R)=(\tau(R))^{2},
$$




$$
\begin{gathered}
u_{1}(R)=\sum_{i, j, h, k} R\left(e_{i}, e_{j}, e_{h}, e_{k}\right) R\left(e_{i}, e_{j}, J e_{h}, J e_{k}\right), \\
u_{2}(R)=\sum_{i, j, h, k} R\left(e_{i}, e_{j}, e_{h}, e_{k}\right) R\left(e_{i}, J e_{j}, e_{h}, J e_{k}\right), \\
u_{3}(R)=\left\langle R, L_{3} R\right\rangle=\sum_{i, j, h, k} R\left(e_{i}, e_{j}, e_{h}, e_{k}\right) R\left(J e_{i}, J e_{j}, J e_{h}, J e_{k}\right), \\
u_{4}(R)=\left\|\rho^{*}(R)\right\|^{2}, \quad u_{5}(R)=\left\langle\rho(R), \rho^{*}(R)\right\rangle, \quad u_{6}(R)=\left\langle\rho(R), \rho\left(L_{3} R\right)\right\rangle, \\
u_{7}(R)=\left\langle\rho^{*}(R), \rho^{*}\left(L_{3} R\right)\right\rangle, \quad u_{8}(R)=\tau(R) \tau^{*}(R), \quad u_{9}(R)=\left(\tau^{*}(R)\right)^{2},
\end{gathered}
$$

where $\left\{e_{i}\right\}$ is an arbitrary orthonormal basis of $V$.

PROOF. The quadratic invariants are of the following type:

$$
P(R)=\sum R\left(e_{i_{1}}, \ldots, e_{i_{4}}\right) R\left(e_{j_{1}}, \ldots, e_{j_{4}}\right) p\left(e_{i_{1}}, \ldots, e_{i_{4}}, e_{j_{1}}, \ldots, e_{j_{4}}\right)
$$

where $p$ is an invariant of $\otimes^{8} V^{*}$. Following the results of [15], $p$ is a linear combination of the $F_{\alpha_{1} \alpha_{2} \alpha_{3} \alpha_{4}}^{\sigma}$, defined as follows:

$$
\begin{aligned}
F_{\alpha_{1} \alpha_{2} \alpha_{3} \alpha_{4}}^{\sigma}\left(x_{1}, \ldots, x_{8}\right)= & \Omega_{\alpha_{1}}\left(x_{\sigma(1)}, x_{\sigma(2)}\right) \Omega_{\alpha_{2}}\left(x_{\sigma(3)}, x_{\sigma(4)}\right) \\
& \cdot \Omega_{\alpha_{3}}\left(x_{\sigma(5)}, x_{\sigma(6)}\right) \Omega_{\alpha_{4}}\left(x_{\sigma(7)}, x_{\sigma(8)}\right),
\end{aligned}
$$

where $\sigma$ is a permutation of $(1, \ldots, 8), \alpha_{i}=0,1$ and $\Omega_{\alpha_{i}}$ is as in $\S 4$.

Because of the symmetries of the curvature tensor $R$, the restrictions of the quadratic invariants to $\mathcal{R}(V)$ are linear combinations of $o_{1}(R), \ldots, u_{9}(R)$.

Using the definitions and properties of $\mho_{3}, W_{6}, \mathcal{W}_{7}$ and $\mathcal{W}_{10}$ we obtain further that the restrictions of the quadratic invariants to these spaces are scalar multiples of $o_{1}(R)$. Hence

THEOREM 6.4. The spaces $\mathscr{W}_{3}, \mathscr{W}_{6}, \mathscr{W}_{7}$ and $\mathscr{W}_{10}$ are irreducible.

Using Theorem 4.4 and Definition 4.6 we obtain with Theorems 6.2 and 6.4 a complete proof of Theorem 5.3(i). The other properties mentioned in Theorem 5.3 will follow at once from Table II in the next section where the dimensions of the subspaces are determined.

We finish this section by determining the linear invariants of $\Re(V)$. (See also [5], [6].) These invariants are the restrictions of linear invariants of $\otimes^{4} V^{*}$, i.e.

$$
P(R)=\sum R\left(e_{i_{1}}, \ldots, e_{i_{4}}\right) p\left(e_{i_{1}}, \ldots, e_{i_{4}}\right)
$$

where $p$ is an invariant of $\otimes^{4} V^{*}$. So $p$ is a linear combination of the $F_{\alpha_{1} \alpha_{2}}^{o}$ we have already used. Taking in account the symmetries of $R \in R(V)$ we obtain

THEOREM 6.5. The space of linear invariants of $R \in \mathcal{R}(V)$ is spanned by $\tau(R)$ and $\tau^{*}(R)$. 
7. Dimensions of the factors. The purpose of this section is to prove

THEOREM 7.1. The dimensions of the factors $W_{i}, i=1, \ldots, 10$, are given in Table II.

\begin{tabular}{|c|c|c|c|}
\hline $\operatorname{dim} V$ & 4 & 6 & $2 n \geqslant 8$ \\
\hline$w_{1}$ & 1 & 1 & 1 \\
\hline$w_{2}$ & 3 & 8 & $n^{2}-1$ \\
\hline $\mathscr{W S}_{3}$ & 5 & 27 & $\frac{1}{4} n^{2}(n-1)(n+3)$ \\
\hline $\mathscr{\mathscr { w } _ { 4 }}$ & 1 & 1 & 1 \\
\hline $\mathscr{w}_{5}$ & 0 & 8 & $n^{2}-1$ \\
\hline $\mathscr{w}_{6}$ & 0 & 0 & $\frac{1}{4} n^{2}(n+1)(n-3)$ \\
\hline $\mathscr{w}_{7}$ & 2 & 12 & $\frac{1}{6} n^{2}\left(n^{2}-1\right)$ \\
\hline था & 6 & 12 & $n(n+1)$ \\
\hline ขึ, & 2 & 6 & $n(n-1)$ \\
\hline$w_{10}$ & 0 & 30 & $\frac{2}{3} n^{2}\left(n^{2}-4\right)$ \\
\hline
\end{tabular}

TABLE II

We shall need two lemmas which we give first. Let $V^{\mathrm{C}}=\mathrm{C} \otimes_{\mathrm{R}} V$. We denote the extension of $J$ to $V^{\mathbf{C}}$ also by $J$, and $V^{+}$, resp. $V^{-}$, are the eigenspaces corresponding to $+i$, resp. $-i$. Hence $V^{\mathrm{C}}=V^{+} \oplus V^{-}$. Now let $R \in R(V)$ and denote by $R^{\mathbf{C}}$ the extension of $R$ to $V^{\mathbf{C}}$. Then $R^{\mathbf{C}}$ is a $\mathbf{C}$-multilinear map with the same symmetry properties as a curvature operator. Moreover, $R^{\mathbf{C}}$ is real, i.e.

$$
R^{\mathrm{C}}(\bar{x}, \bar{y}, \bar{z}, \bar{w})=\overline{R^{\mathrm{C}}(x, y, z, w)} .
$$

Then we have

LeMma 7.2. The real vector space $\Re_{3}^{\perp}(V)$ is isomorphic with the space of $\mathbf{C}$-multilinear maps $L: V^{+} \times V^{+} \times V^{+} \times V^{-} \rightarrow \mathbf{C}$ satisfying

$$
\begin{gathered}
L(x, y, z, \bar{w})=-L(y, x, z, \bar{w}), \\
L(x, y, z, \bar{w})+L(y, z, x, \bar{w})+L(z, x, y, \bar{w})=0
\end{gathered}
$$

for all $x, y, z, w \in V^{\mathbf{c}}$.

Proof. $R \in \mathscr{R}_{3}^{\perp}(V)$ if and only if $R(x, y, z, w)=-R(J x, J y, J z, J w)$. The same property is valid for the extension $R^{\mathrm{C}}$. Hence $R^{\mathrm{C}}(x, y, z, w)=0$ when $x, y, z, w \in$ $V^{+}$(resp. $V^{-}$) or when two of them belong to $V^{+}$and the two others to $V^{-}$. So, the only nonvanishing terms are of type $R^{\mathrm{C}}(x, y, z, \bar{w})$ where $x, y, z, w \in V^{+}$or are obtained from those by conjugation or the symmetry properties, including the Bianchi identity. Hence, when we denote by $\phi(R)$ the restriction of $R^{\mathrm{C}}$ to $V^{+} \oplus V^{+} \oplus V^{+} \oplus V^{-}$, we obtain the required isomorphism $\phi$. 
LEMMA 7.3. The real space $\mho_{7}=\Re_{2}^{\perp}(V)$ is isomorphic with the space of $\mathbf{C}$-multilinear maps $L: V^{+} \times V^{+} \times V^{+} \times V^{+} \rightarrow \mathrm{C}$ which satisfies all the symmetry properties of the curvature operators.

Proof. For $R \in \Re_{2}^{\perp}(V)$, Lemma 3.8 implies $R^{\mathrm{C}}(x, y, z, w)=0$ when at least one of the vectors belongs to $V^{+}$and at least one to $V^{-}$. Hence the only nonvanishing terms are of type $R^{\mathrm{C}}(x, y, z, w), x, y, z, w \in V^{+}$, or $R^{\mathrm{C}}(\bar{x}, \bar{y}, \bar{z}, \bar{w})$. As in Lemma 7.2 we again take the restriction of $R^{\mathrm{C}}$ to $V^{+} \times V^{+} \times V^{+} \times V^{+}$to obtain the required isomorphism.

Proof of Theorem 7.1. Theorem 4.4 and Definition 4.6 imply that dim $\mathscr{w}_{1}=$ $\operatorname{dim} \mathscr{W}_{4}=1$.

Next using Theorem 5.7, the remark above Theorem 6.2, Theorem 6.2 and (6.2), we get

$$
\begin{aligned}
& \operatorname{dim} \mathscr{W}_{2}=n^{2}-1, \quad \operatorname{dim} \mathscr{W}_{5}= \begin{cases}0 & \text { for } n=2, \\
n^{2}-1 & \text { for } n \geqslant 3,\end{cases} \\
& \operatorname{dim} \mathscr{W}_{8}=n(n+1), \quad \operatorname{dim} \mathscr{W}_{9}=n(n-1) .
\end{aligned}
$$

Further, in [22] it is proved that

$$
\operatorname{dim} \mathscr{W}_{3}=\frac{1}{4} n^{2}(n-1)(n+3) .
$$

Hence we still need to compute the dimensions of $\mathscr{w}_{6}, \mathscr{W}_{7}$ and $\mathcal{W}_{10}$. Therefore we only need to know $\operatorname{dim}\left(\mathscr{W}_{7}=\Re_{2}^{\perp}(V)\right)$ and $\operatorname{dim} \Re_{3}^{\perp}(V)$ (= $\operatorname{dim} \mathscr{W}_{8} \oplus \mathscr{W}_{9} \oplus$ $\left.\mathscr{W}_{10}\right)$ since it is well known that

$$
\operatorname{dim} \Re(V)=(\operatorname{dim} V)^{2}\left\{\left(\operatorname{dim} V^{2}\right)-1\right\} / 12=\frac{1}{3} n^{2}\left(4 n^{2}-1\right) .
$$

But Lemma 7.2 implies

$$
\operatorname{dim} \Re_{3}^{\perp}(V)=\frac{2}{3} n^{2}\left(n^{2}-1\right)
$$

because the space of the $\mathbf{C}$-multilinear maps satisfying only the first condition is $n^{2}\left(\begin{array}{l}n \\ 2\end{array}\right)$-dimensional. The second condition gives $n\left(\begin{array}{l}n \\ 3\end{array}\right)$ linear independent conditions.

In a similar way one obtains

$$
\operatorname{dim} \Re_{2}^{\perp}(V)=\frac{1}{6} n^{2}\left(n^{2}-1\right) .
$$

So we have the results of Table III:

\begin{tabular}{|c|c|c|c|}
\hline $\operatorname{dim} V$ & 4 & 6 & $2 n>8$ \\
\hline $\operatorname{dim} \Re_{1}(V)$ & 9 & 36 & $\frac{1}{4} n^{2}(n+1)^{2}$ \\
\hline $\operatorname{dim} \Re_{2}(V)$ & 10 & 45 & $\frac{1}{2} n^{2}\left(n^{2}+1\right)$ \\
\hline $\operatorname{dim} \Re_{3}(V)$ & 12 & 57 & $\frac{1}{3} n^{2}\left(2 n^{2}+1\right)$ \\
\hline $\operatorname{dim} \Re_{(V)}$ & 20 & 105 & $\frac{1}{3} n^{2}\left(4 n^{2}-1\right)$ \\
\hline
\end{tabular}

TABLE III

This gives the required result. 
8. Projections and norms. In this section we write down the explicit formulas for the projections of an element $R \in \mathcal{R}(V)$ on the irreducible factors $\mho_{i}$ and compute the norms of the projections.

Theorem 8.1. Let $R \in \Re(V), \operatorname{dim} V=2 n \geqslant 8$, and denote by $p_{i}, i=1, \ldots, 10$, the projection operators of $R(V)$ on $\mho_{i}$. Then we have:

$$
\begin{aligned}
& p_{1}(R)=\frac{\tau(R)+3 \tau^{*}(R)}{16 n(n+1)}\left(\pi_{1}+\pi_{2}\right) \\
& p_{2}(R)=\frac{1}{16}(n+2)^{-1}(\varphi+\psi)\left\{\rho\left(R+L_{3} R\right)+3 \rho^{*}\left(R+L_{3} R\right)\right. \\
& \left.-n^{-1}\left(\tau(R)+3 \tau^{*}(R)\right) g\right\} \\
& p_{3}(R)=\frac{1}{8}\left(I+L_{1}\right)\left(I+L_{2}\right)\left(I+L_{3}\right) R-p_{1}(R)-p_{2}(R) \text {; } \\
& p_{4}(R)=\frac{\tau(R)-\tau^{*}(R)}{16 n(n-1)}\left(3 \pi_{1}-\pi_{2}\right) ; \\
& p_{5}(R)=\frac{1}{16}(n-2)^{-1}(3 \varphi-\psi)\left\{\rho\left(R+L_{3} R\right)-\rho^{*}\left(R+L_{3} R\right)\right. \\
& \left.-n^{-1}\left(\tau(R)-\tau^{*}(R)\right) g\right\} \\
& p_{6}(R)=\frac{1}{8}\left(I-L_{1}\right)\left(I+L_{2}\right)\left(I+L_{3}\right) R-p_{4}(R)-p_{5}(R) \text {; } \\
& p_{7}(R)=\frac{1}{4}\left(I-L_{2}\right)\left(I+L_{3}\right) R ; \quad p_{8}(R)=\frac{1}{4}(n-1)^{-1} \varphi\left(\rho\left(R-L_{3} R\right)\right) ; \\
& p_{9}(R)=\frac{1}{4}(n+1)^{-1} \psi\left(\rho^{*}\left(R-L_{3}\right)\right) ; \quad p_{10}(R)=\frac{1}{2}\left(I-L_{3}\right) R-p_{8}(R)-p_{9}(R),
\end{aligned}
$$

where I denotes the identity transformation of $V$.

Proof. We will compute, for example, $p_{4}(R), p_{5}(R)$ and $p_{6}(R)$. The other projections can be obtained by a similar procedure.

Let $R \in \mathscr{W}_{4} \oplus \mathscr{W}_{5} \oplus \mathscr{W}_{6}$. Using (3.3), Theorem 3.6 and Definition 5.1, we get

$$
p_{4}(R)+p_{5}(R)+p_{6}(R)=\frac{1}{8}\left(I-L_{1}\right)\left(I+L_{2}\right)\left(I+L_{3}\right) R \text {. }
$$

But

$$
p_{4}(R)=\lambda\left(3 \pi_{1}-\pi_{2}\right) \text { and } \tau\left(p_{5}(R)\right)=\tau\left(p_{6}(R)\right)=0
$$

Hence

$$
\lambda \tau\left(3 \pi_{1}-\pi_{2}\right)=\frac{1}{8} \tau\left(\left(I-L_{1}\right)\left(I+L_{2}\right)\left(I+L_{3}\right) R\right) .
$$

Using Lemmas 3.9-3.11 we get

$$
\lambda=\left(\tau(R)-\tau^{*}(R)\right) / 16 n(n-1) .
$$

Further, we also have

$$
p_{5}(R)=(3 \varphi-\psi)(S), \quad S \in \mathscr{V}_{1}, \quad \text { and } \quad \rho\left(p_{6}(R)\right)=0 .
$$

So, using Lemmas 3.10, 3.11, 3.15, 5.8 and 5.9 we obtain

$$
\begin{aligned}
6(n-1) \lambda g+6(n-2) S & =\frac{1}{8} \rho\left(\left(I-L_{1}\right)\left(I+L_{2}\right)\left(I+L_{3}\right) R\right) \\
& =\frac{3}{16} \rho\left(\left(I+L_{2}\right)\left(I+L_{3}\right) R\right)-\frac{3}{16} \rho^{*}\left(\left(I+L_{2}\right)\left(I+L_{3}\right) R\right) \\
& =\frac{3}{8}\left\{\rho\left(R+L_{3} R\right)-\rho^{*}\left(R+L_{3} R\right)\right\},
\end{aligned}
$$

which gives the required expression for $S$. 
The square of the length of $p_{i}(R)$ is a quadratic invariant. Hence it must be a linear combination of the 12 invariants determined in $\$ 6$. We have explicitly

THeOREM 8.2. Let $R \in \mathcal{R}(V)$. Then for $n \geqslant 2$ the $\left\|p_{i}(R)\right\|^{2}, i=1, \ldots, 10$, are given by

$$
\begin{aligned}
\left\|p_{1}(R)\right\|^{2}= & \frac{1}{8 n(n+1)}\left\{\tau(R)+3 \tau^{*}(R)\right\}^{2}=\frac{1}{8 n(n+1)}\left(o_{3}+6 u_{8}+9 u_{9}\right)(R) ; \\
\left\|p_{2}(R)\right\|^{2}= & \frac{1}{4(n+2)}\left\{o_{2}+u_{6}+9 u_{4}+9 u_{7}+12 u_{5}-n^{-1}\left(o_{3}+6 u_{8}+9 u_{9}\right)\right\}(R) ; \\
\left\|p_{3}(R)\right\|^{2}= & \left\{\frac{1}{16}\left(3 o_{1}+10 u_{1}-4 u_{2}+3 u_{3}\right)\right. \\
& -\frac{1}{4(n+2)}\left(o_{2}+u_{6}+9 u_{4}+9 u_{7}+12 u_{5}\right) \\
& \left.+\frac{1}{8(n+1)(n+2)}\left(o_{3}+6 u_{8}+9 u_{9}\right)\right\}(R) ; \\
\left\|p_{4}(R)\right\|^{2}= & \frac{3}{8 n(n-1)}\left\{\tau(R)-\tau^{*}(R)\right\}^{2}=\frac{3}{8 n(n-1)}\left(o_{3}-2 u_{8}+u_{9}\right)(R) ; \\
\left\|p_{5}(R)\right\|^{2}= & \frac{3}{4(n-2)}\left\{o_{2}+u_{6}+u_{4}+u_{7}-4 u_{5}-n^{-1}\left(o_{3}-2 u_{8}+u_{9}\right)\right\}(R) ; \\
\left\|p_{6}(R)\right\|^{2}= & \left\{\frac{3}{16}\left(o_{1}-2 u_{1}+4 u_{2}+u_{3}\right)\right. \\
& -\frac{3}{4(n-2)}\left(o_{2}+u_{6}+u_{4}+u_{7}-4 u_{5}\right) \\
\left\|p_{7}(R)\right\|^{2}= & \frac{1}{8}\left(o_{1}-2 u_{1}-4 u_{2}+u_{3}\right)(R) ; \\
\left\|p_{8}(R)\right\|^{2}= & (n-1)^{-1}\left(o_{2}-u_{6}\right)(R) ; \\
\left\|p_{9}(R)\right\|^{2}= & (n+1)^{-1}\left(u_{4}-u_{7}\right)(R) ; \\
\left\|p_{10}(R)\right\|^{2}= & \left\{\frac{1}{2}\left(o_{1}-u_{3}\right)-(n-1)^{-1}\left(o_{2}-u_{6}\right)-(n+1)^{-1}\left(u_{4}-u_{7}\right)\right\}(R)
\end{aligned}
$$

Proof. $\left\|p_{i}(R)\right\|^{2}, i=1,2,4,5,8$ and 9 , follows after some calculations using Lemmas 5.8, 5.9 and Theorem 5.14.

Next, since the decomposition is orthogonal we have

$$
\begin{aligned}
\left\|\sum_{i=8}^{10} p_{i}(R)\right\|^{2} & =\sum_{i=8}^{10}\left\|p_{i}(R)\right\|^{2}=\frac{1}{4}\left\|R-L_{3} R\right\|^{2} \\
& =\frac{1}{2}\left\{\|R\|^{2}-\left\langle R, L_{3} R\right\rangle\right\}=\frac{1}{2}\left(o_{1}-u_{3}\right)(R) .
\end{aligned}
$$

Hence one can compute $\left\|p_{10}(R)\right\|^{2}$.

Further, we have

$$
p_{7}(R)=\frac{1}{4}\left(I-L_{2}\right)\left(I+L_{3}\right) R
$$


and so

$$
\left\|p_{7}(R)\right\|^{2}=\frac{1}{8}\left\{\left\|R+L_{3} R\right\|^{2}-\left\langle\left(I+L_{3}\right) R, L_{2}\left(I+L_{3}\right) R\right\rangle\right\} .
$$

Now put $\tilde{R}=R+L_{3} R$. Then $\tilde{R} \in \mathscr{R}_{3}(V)$ and so $2\left\langle\tilde{R}, L_{2} \tilde{R}\right\rangle=o_{1}(\tilde{R})+u_{1}(\tilde{R})$ $+2 u_{2}(\tilde{R})$. Direct computation gives

$$
\begin{gathered}
o_{1}(\tilde{R})=o_{1}\left(R+L_{3} R\right)=2 o_{1}(R)+2 u_{3}(R), \\
u_{1}(\tilde{R})=u_{1}\left(R+L_{3} R\right)=4 u_{1}(R), \quad u_{2}(\tilde{R})=4 u_{2}(R) .
\end{gathered}
$$

This gives the required result for $\left\|p_{7}(R)\right\|^{2}$.

Now it is possible to compute $\sum_{i=1}^{6}\left\|p_{i}(R)\right\|^{2}$. To obtain all the norms we still have to compute the norm of

$$
\sum_{i=1}^{3} p_{i}(R)=\frac{1}{8}\left(I+L_{1}\right)\left(I+L_{2}\right)\left(I+L_{3}\right) R .
$$

We omit the long calculation.

THEOREM 8.3. The formulas of Theorems 8.1 and 8.2 are still valid for $n=3$ and $n=2$. Moreover, for $n=3$ we have $p_{6}(R)=0$, and, for $n=2, p_{5}(R)=p_{6}(R)=$ $p_{10}(R)=0$.

9. Weyl and Bochner tensors. Let $V$ be an arbitrary real vector space with $\operatorname{dim} V=m$. We recall that the Weyl component $\Re_{W}$ of $\Re(V)$ is formed by the curvature tensors $R$ with vanishing Ricci tensor [21], [18]. Tensors belonging to $\mathcal{R}_{W}(V)$ are called Weyl tensors. The Weyl tensor $C(R)$ associated with $R$ is the projection of $R$ on $\Re_{W}(V)$. We have

$$
\begin{aligned}
C(R)(x, y) z & =R(x, y) z \\
& -(m-2)^{-1}\{g(x, z) Q y-g(y, z) Q x+g(Q x, z) y-g(Q y, z) x\} \\
+ & \{\tau(R) /(m-1)(m-2)\}\{g(x, z) y-g(y, z) x\} .
\end{aligned}
$$

Note that $C(R)=0$ for $m=3$.

It is worthwhile to note that the orthogonal complement of $\Re_{W}(V)$ in $\Re(V)$ is the subspace spanned by the curvature tensors of type $\varphi(S)$ where $S$ is symmetric.

Now we return again to a Hermitian vector space $V$ of real dimension $2 n$ and determine the Weyl component. Using Theorem 5.3 and Lemmas 5.8-5.9 we get

THEOREM 9.1. The decomposition of the Weyl component $\Re_{W}(V)$ of $R(V)$ into orthogonal irreducible factors for the action of $\mathcal{U}(n)$ is given by:

(i) $\Re_{W}(V)=\mathfrak{L}\left(3 \pi_{1}-(2 n-1) \pi_{2}\right) \oplus(3 \varphi-(n-1) \psi)\left(\mathscr{V}_{1}\right)$ $\oplus \mathcal{W}_{3} \oplus \mathcal{W}_{6} \oplus \mathcal{W}_{7} \oplus \mathcal{W}_{9} \oplus \mathcal{W}_{10}$, for $n \geqslant 4$;

(ii) $\Re_{W}(V)=\mathfrak{L}\left(\pi_{1}-\pi_{2}\right) \oplus \mho_{3} \oplus W_{7} \oplus W_{9}$, for $n=2$;

(iii) $\mathscr{R}_{W}(V)=\mathfrak{L}\left(3 \pi_{1}-5 \pi_{2}\right) \oplus(3 \varphi-2 \psi)\left(\mathcal{V}_{1}\right) \oplus \mathcal{W}_{3} \oplus \mathcal{W}_{7} \oplus \mathcal{W}_{9} \oplus \mathcal{W}_{10}$, for $n=3$.

Since any symmetric tensor $S \in \otimes^{2} V^{*}$ may be written as $S=\lambda g+S_{1}+S_{2}$ where $S_{1} \in \mathfrak{V}_{1}$ and $S_{2} \in \mathfrak{T}_{2}$, we have

THEOREM 9.2. $\mathcal{R}_{W}^{\perp}(V)=\mathcal{L}\left(\pi_{1}\right) \oplus \varphi\left(\mathscr{V}_{1}\right) \oplus \varphi\left(\mathfrak{V}_{2}\right)$. 
The theory for the Bochner tensor $B(R)$ associated with a curvature tensor $R \in \Re_{1}(V)$ is now well known [16], [17], [22]. We now generalize this notion for arbitrary curvature tensors $R \in \Re(V)$. This generalization is quite natural since it is based on the vanishing of the two basic $\mathcal{U}(n)$-concomitants $\rho$ and $\rho^{*}$. (For $R \in \mathcal{R}_{1}, \rho$ and $\rho^{*}$ coincide.) Further, it will turn out that $B(R)$ is a conformal invariant tensor which has a lot of properties in common with the Bochner tensor for curvature tensors belonging to $\Re_{1}(V)$ (see, for example, $\S 15$ ). Finally, in $\S 14$ we will show that for $n=2$ there is a nice relation with the anti-self-dual part of the Weyl conformal tensor.

DEFINITION 9.3. The Bochner component $\Re_{B}(V)$ of $\Re(V)$ is the subspace of $\Re_{W}(V)$ formed by the curvature tensors $R \in \mathcal{R}(V)$ such that $\rho^{*}(R)=0$.

A tensor belonging to $\Re_{B}(V)$ is called a Bochner tensor and the projection $B(R)$ of $R \in \mathcal{R}(V)$ on $\Re_{B}(V)$ is called the Bochner conformal tensor associated with $R$.

We will justify the notion "conformal” in $\S 11$.

We have, using Theorem 8.1

THEOREM 9.4. Let $R \in \mathcal{R}(V)$. Then the Bochner component is given by

$$
\Re_{B}(V)=\psi_{3} \oplus \psi_{6} \oplus W_{7} \oplus W_{10}
$$

and the Bochner conformal tensor associated with $R$ by

$$
\begin{aligned}
B(R)= & R-\{16(n+2)\}^{-1}(\varphi+\psi)\left(\rho+3 \rho^{*}\right)\left(R+L_{3} R\right) \\
& -\{16(n-2)\}^{-1}(3 \varphi-\psi)\left(\rho-\rho^{*}\right)\left(R+L_{3} R\right) \\
& -\left\{\{4(n+1)\}^{-1}\left(\psi \circ \rho^{*}\right)+\{4(n-1)\}^{-1}(\varphi \circ \rho)\right\}\left(R-L_{3} R\right) \\
& +\{16(n+1)(n+2)\}^{-1}\left(\tau+3 \tau^{*}\right)(R)\left(\pi_{1}+\pi_{2}\right) \\
& +\{16(n-1)(n-2)\}^{-1}\left(\tau-\tau^{*}\right)(R)\left(3 \pi_{1}-\pi_{2}\right)
\end{aligned}
$$

for $n \geqslant 3$, and by

$$
\begin{aligned}
B(R)= & R-\frac{1}{2} \varphi\left(\rho(R)-\frac{1}{4} \tau(R) g\right)-\frac{1}{12} \psi\left(\rho^{*}\left(R-L_{3} R\right)\right) \\
& -\frac{1}{96}\left(\tau+3 \tau^{*}\right)(R)\left(\pi_{1}+\pi_{2}\right)-\frac{1}{32}\left(\tau-\tau^{*}\right)(R)\left(3 \pi_{1}-\pi_{2}\right)
\end{aligned}
$$

for $n=2$.

We note that for $R \in \mathscr{R}_{1}(V)$ we get

$$
B(R)=R-\frac{1}{2(n+2)}(\varphi+\psi)(\rho(R))+\frac{1}{4(n+1)(n+2)} \tau(R)\left(\pi_{1}+\pi_{2}\right),
$$

which is the usual Bochner tensor.

Using this theorem and Theorem 8.2 we obtain

THEOREM 9.5. Let $B(R)$ be the Bochner conformal tensor associated with $R \in$ $\Re(V)$. Then we have

$$
\begin{aligned}
\|B(R)\|^{2}=\{ & o_{1}-(n+1)\left(n^{2}-4\right)^{-1}\left(o_{2}+u_{6}\right)-(n-1)^{-1}\left(o_{2}-u_{6}\right) \\
& -3(n-1)\left(n^{2}-4\right)^{-1}\left(u_{4}+u_{7}\right) \\
& -(n+1)^{-1}\left(u_{4}-u_{7}\right)+\{8(n+1)(n+2)\}^{-1} \\
& \left.\cdot\left(o_{3}+6 u_{8}+9 u_{9}\right)+3\{8(n-1)(n-2)\}^{-1}\left(o_{3}-6 u_{8}+u_{9}\right)\right\}(R)
\end{aligned}
$$


for $n \geqslant 3$, and

$$
\begin{aligned}
& \|B(R)\|^{2} \\
& \quad=\left\{o_{1}-2 o_{2}+8\left(u_{4}-u_{7}\right)-\frac{1}{48}\left(o_{3}+6 u_{8}+9 u_{9}\right)-\frac{3}{16}\left(o_{3}-2 u_{8}+u_{9}\right)\right\}(R)
\end{aligned}
$$

for $n=2$.

10. Almost Hermitian manifolds. First applications. Let $(M, g, J)$ be an almost Hermitian manifold with real dimension $2 n$, Riemannian connection $\nabla$ and curvature tensor $R$ given by

$$
R(X, Y)=\nabla_{[X, Y]}-\left[\nabla_{X}, \nabla_{Y}\right]
$$

where $X, Y \in \mathcal{X}(M)$, the algebra of $C^{\infty}$ vector fields on $M$.

Let $T_{m} M, m \in M$, be the tangent space at $m$ with complex structure $J_{m}$ and inner product $g_{m}$. We denote by $\mathcal{R}(M)$ the vector bundle with fibre $\Re\left(T_{m} M\right)$. The decomposition (5.1) for $\Re\left(T_{m} M\right)$ gives rise to a decomposition of $\mathscr{R}(M)$ in subbundles, orthogonal with respect to the fibre metric of $R(M)$ induced by $g$. We still denote the components of the decomposition by $\mho_{i}, i=1, \ldots, 10$.

The Riemann curvature tensor on $M$ is a section of $\Re(M)$ and, in general, we do not have a particular identity which is fulfilled by $R$. The two following theorems are derived from Theorem 5.3 and give curvature identities on 4 - and 6-dimensional almost Hermitian manifolds.

THEOREM 10.1. Let $M$ be an almost Hermitian manifold with real dimension 4 and curvature tensor $R$. Then we have the following identities:

$$
\begin{gathered}
\left(I-L_{1}\right)\left(I+L_{2}\right)\left(I+L_{3}\right) R=\frac{1}{4}\left(\tau(R)-\tau^{*}(R)\right)\left(3 \pi_{1}-\pi_{2}\right) \\
R-L_{3} R=\frac{1}{2} \varphi\left(\rho\left(R-L_{3} R\right)\right)+\frac{1}{6} \psi\left(\rho^{*}\left(R-L_{3} R\right)\right) \\
\rho\left(R+L_{3} R\right)-\rho^{*}\left(R+L_{3} R\right)=\frac{1}{2}\left(\tau(R)-\tau^{*}(R)\right) g .
\end{gathered}
$$

THEOREM 10.2. Let $M$ be an almost Hermitian manifold with real dimension 6 and curvature tensor $R$. Then we have the following identity:

$$
\begin{aligned}
\left(I-L_{1}\right)\left(I+L_{2}\right)\left(I+L_{3}\right) R= & \frac{1}{2}(3 \varphi-\psi)\left\{\rho\left(R+L_{3} R\right)-\rho^{*}\left(R+L_{3} R\right)\right\} \\
& -\frac{1}{4}\left(\tau(R)-\tau^{*}(R)\right)\left(3 \pi_{1}-\pi_{2}\right) .
\end{aligned}
$$

These identities are still valid on an arbitrary-dimensional Kähler manifold since then $L_{i} R=R, i=1,2,3 ; \rho(R)=\rho^{*}(R)$ and $\tau(R)=\tau^{*}(R)$.

Since curvature identities are a key to understanding the geometry of an almost Hermitian manifold, it is interesting to study classes of almost Hermitian manifolds with Riemann curvature tensor belonging to a particular subclass of $\Re(V)$.

Definition 10.3 [10], [12].

$Q \mathcal{F}_{i}=\{$ almost Hermitian manifolds $M$ with Riemann

$$
\text { curvature tensor } \left.R \in \Re_{i}\left(T_{m} M\right) \text { for all } m \in M\right\}, \quad i=1,2,3 \text {. }
$$


We are at this stage able to characterize these classes using the quadratic invariants. Indeed, we obtain from Theorem 8.2:

THEOREM 10.4. Let $M$ be an almost Hermitian manifold with Riemann curvature tensor $R$. Then,

(i) $o_{1}(R) \geqslant u_{3}(R)$ with equality sign if and only if $M \in \mathbb{Q} \mathcal{F}_{3}$;

(ii) $5 o_{1}(R) \geqslant\left(2 u_{1}+4 u_{2}+3 u_{3}\right)(R)$ with equality sign if and only if $M \in \mathbb{Q} \mathcal{K}_{2}$;

(iii) $13 o_{1}(R) \geqslant\left(10 u_{1}-4 u_{2}+3 u_{3}\right)(R)$ with equality sign if and only if $M \in$ $Q \mathcal{H}_{1}$.

11. Conformal invariants. On an arbitrary almost Hermitian manifold $(M, J, g)$ we now consider the following conformal change of metric: $\tilde{g}=e^{\sigma} g$ where $\sigma$ is a $C^{\infty}$ function on $M$. Let $\tilde{R}$ denote the Riemann tensor associated with $\tilde{g}$. Then we have the following well-known relation between $\tilde{R}$ and $R$ :

$$
\begin{aligned}
e^{-\sigma} \tilde{R}(X, Y, Z, W)= & R(X, Y, Z, W) \\
& -\frac{1}{2}\{L(X, Z) g(Y, W)+L(Y, W) g(X, Z) \\
& -L(X, W) g(Y, Z)-L(Y, Z) g(X, W)\} \\
& -\left(\|\omega\|^{2} / 4\right)\{g(X, Z) g(Y, W)-g(Y, Z) g(X, W)\}
\end{aligned}
$$

where

$$
\omega=d \sigma, \quad L(X, Y)=\left(\nabla_{X} \omega\right)(Y)-\frac{1}{2} \omega(X) \omega(Y)
$$

and $X, Y, Z, W \in \mathfrak{X}(M)$. Note that $L$ is symmetric.

We put

$$
T_{\sigma}=-\frac{1}{2} \varphi(L)-\left(\|\omega\|^{2} / 4\right) \pi_{1}
$$

By doing this, (11.1) becomes

$$
e^{-\sigma} \tilde{R}=R+T_{\sigma}
$$

The curvature tensor $T_{\sigma}$ is orthogonal to the Weyl component $\Re_{W}(V)$ and, hence, $C\left(T_{\sigma}\right)=0$.

Next we consider $\mathcal{R}_{W}(M)$ and define the following subbundles (see Theorem 9.1):

$$
\begin{aligned}
& \mathcal{C}_{1}(M)=\mathcal{L}\left(3 \pi_{1}-(2 n-1) \pi_{2}\right), \quad \varrho_{2}(M)=(3 \varphi-(n-1) \psi)\left(\mathscr{V}_{1}\right), \quad \bigodot_{3}(M)=\mathcal{W}_{3}, \\
& e_{4}(M)=\psi_{6}, \quad e_{5}(M)=\psi_{7}, \quad e_{6}(M)=\psi_{9}, \quad e_{7}(M)=\psi_{10} \text {. }
\end{aligned}
$$

Further, let $C_{i}(R)$ denote the projection of $R$ on $\bigodot_{i}(M)$. Then we have

$$
C(R)=\sum_{i=1}^{7} C_{i}(R), \quad B(R)=C_{3}(R)+C_{4}(R)+C_{5}(R)+C_{7}(R) .
$$

Since $C\left(T_{\sigma}\right)=0$, we obtain from $(11.3)$

THEOREM 11.1. $C_{i}(R), i=1, \ldots, 7$, and hence $C(R)$ and $B(R)$ are conformally invariant. 
The explicit expressions for $C_{i}(R), i=3, \ldots, 7$, and their norms are given in Theorems 8.1 and 8.2. Further, we easily find

THEOREM 11.2.

$$
\begin{aligned}
& C_{1}(R)=\left\{8 n\left(n^{2}-1\right)(2 n-1)\right\}^{-1}\left\{\tau-(2 n-1) \tau^{*}\right\}(R)\left\{3 \pi_{1}-(2 n-1) \pi_{2}\right\}, \\
& C_{2}(R)=(n-1)^{-1}\{3 \varphi-(n-1) \psi\}\left(S_{2}-S_{1}\right)
\end{aligned}
$$

where

$$
\begin{aligned}
& 16(n+2) S_{1}=\left(\rho+3 \rho^{*}\right)\left(R+L_{3} R\right)-n^{-1}\left(\tau+3 \tau^{*}\right)(R) g, \\
& 16(n-2) S_{2}=\left(\rho-\rho^{*}\right)\left(R+L_{3} R\right)-n^{-1}\left(\tau-\tau^{*}\right)(R) g
\end{aligned}
$$

for $n \geqslant 3$ and $C_{2}(R)=0$ for $n=2$. Moreover,

$$
\begin{aligned}
\left\|C_{1}(R)\right\|^{2} & =\left\{3 / 4 n\left(n^{2}-1\right)(2 n-1)\right\}\left\{\tau(R)-(2 n-1) \tau^{*}(R)\right\}^{2} \\
& =\left\{3 / 4 n\left(n^{2}-1\right)(2 n-1)\right\}\left\{o_{3}-2(2 n-1) u_{8}+(2 n-1)^{2} u_{9}\right\}(R)
\end{aligned}
$$

and

$$
\begin{aligned}
\left\|C_{2}(R)\right\|^{2}=\left\{3 /\left(n^{2}-4\right)(n-1)\right\} & \\
& \cdot\left\{o_{2}+(n-1)^{2} u_{4}-4(n-1) u_{5}+u_{6}+(n-1)^{2} u_{7}\right. \\
& \left.-n^{-1}\left(o_{3}-2(n-1) u_{8}+(n-1)^{2} u_{9}\right)\right\}(R)
\end{aligned}
$$

for $n \geqslant 3$.

Some of the $C_{i}(R)$ vanish on manifolds belonging to special classes of almost Hermitian manifolds. We give some examples. First of all we have

THEOREM 11.3. Let $R$ be an arbitrary curvature tensor field on a general almost Hermitian manifold of complex dimension $n$. Then:

(i) $C_{2}(R)=C_{4}(R)=C_{7}(R)=0$ for $n=2$;

(ii) $C_{4}(R)=0$ for $n=3$.

Next we consider a Hermitian manifold $M$. A. Gray proved in [10] that the Riemann curvature tensor $R$ of $M$ satisfies the following identity:

$$
\begin{aligned}
R(X, Y, Z, W)+ & R(J X, J Y, J Z, J W) \\
= & R(J X, J Y, Z, W)+R(X, Y, J Z, J W)+R(J X, Y, J Z, W) \\
& +R(X, J Y, Z, J W)+R(J X, Y, Z, J W)+R(X, J Y, J Z, W) .
\end{aligned}
$$

This is equivalent to the condition $R+L_{3} R=L_{2}\left(R+L_{3} R\right)$. Hence

THEOREM 11.4. Let $R$ be the Riemann curvature tensor of a Hermitian manifold $M$. Then $C_{5}(R)=0$.

In [24] we gave an example of a 4-dimensional manifold such that $C_{5}(R)=0$, but which is not Hermitian.

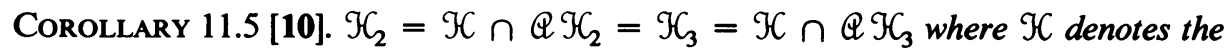
class of Hermitian manifolds. 
Finally we consider the class of Hermitian manifolds with are locally conformal to a Kähler manifold. (See [26], [27].) Since $C_{4}(R)=C_{5}(R)=C_{6}(R)=C_{7}(R)=0$ for Kähler manifolds, we have from this and Theorem 11.1:

THEOREM 11.6. Let $M$ be a locally conformal Kähler manifold. Then $C_{i}(R)=0$ for $i=4,5,6,7$.

These conditions are only necessary conditions. Indeed, consider the manifold $M=M^{2} \times \mathbf{R}^{4}$ where $M^{2}$ is a minimal surface in $\mathbf{R}^{3}$. It is proved in [10] that $M$ is a Hermitian manifold such that $L_{3} R=R$. This implies $C_{5}(R)=C_{6}(R)=C_{7}(R)=$ 0 and, since $\operatorname{dim} M=6$, we also have $C_{4}(R)=0$. On the other hand $M$ is not a locally conformal Kähler manifold [11].

We finish this section with

THEOREM 11.7. Let $M$ be a locally conformal Kähler manifold with $\operatorname{dim} M=2 n \geqslant$ 6 and $C_{2}(R)=C_{3}(R)=0$. Then $M$ is locally conformal to $\mathbf{C} P^{n}(\mu), \mathbf{C} D^{n}(\mu)$ or $\mathbf{C}^{n}$. When $\mu \neq 0, M$ is a globally conformal Kähler manifold.

Proof. By Theorem 11.1 $M$ is locally conformal to a Kähler manifold $\tilde{M}$ such that $C_{2}(\tilde{R})=C_{3}(\tilde{R})=0$. But, since $\operatorname{dim} \tilde{M} \geqslant 6$ and

$$
C_{2}(\tilde{R})=\{-1 / 2(n-1)(n+2)\}(3 \varphi-(n-1) \psi)(\rho(\tilde{R})-(\tau(\tilde{R}) / 2 n) g),
$$

$C_{2}(\tilde{R})=0$ if and only if $\rho(\tilde{R})=(\tau(\tilde{R}) / 2 n) g$. Hence, if $C_{3}(\tilde{R})=0, \tilde{M}$ has constant holomorphic sectional curvature. The rest follows from a result in [27].

12. Generalized complex space forms. The decomposition of $\Re(V)$ is very useful to derive inequalities for the quadratic invariants and to characterize some special classes of manifolds $M$. A well-known example is $\|\rho(R)\|^{2}>(1 / 2 n)(\tau(R))^{2}$, where the equality occurs if and only if $M$ is an Einstein manifold, i.e. $\rho(R)=$ $(\tau(R) / 2 n) g$. Note that the subspace of $R(V)$ formed by the Einstein curvature tensors is given by the subspace where $\varphi\left(\mathscr{V}_{1}\right)=\varphi\left(\mathscr{V}_{2}\right)=0$ (see Theorem 9.2). We note further that $\varphi\left(\mathscr{V}_{2}\right)=\mathcal{W}_{8}$ and $\varphi\left(\mathscr{V}_{1}\right)$ is the subspace of $\mathscr{W}_{2} \oplus \mathcal{W}_{5}$ which is orthogonal to $(3 \varphi-(n-1) \psi)\left(\mathscr{V}_{1}\right)$.

We consider now a similar case.

Definition 12.1. A manifold $(M, J, g)$ is said to be *-Einsteinian if and only if $\rho^{*}(R)=\left(\tau^{*}(R) / 2 n\right) g$.

Using the Cauchy-Schwarz inequality or the decomposition (5.1) it is easy to prove

THEOREM 12.2. We have $\left\|\rho^{*}(R)\right\|^{2} \geqslant(1 / 2 n)\left(\tau^{*}(R)\right)^{2}$, where equality occurs if and only if $M$ is *-Einsteinian.

A particular class of manifolds which are Einsteinian and *-Einsteinian has been considered in [28]-[30]. There we considered the so-called generalized complex space forms. They are defined as being manifolds $M \in \mathbb{Q} \mathcal{H}_{3}$ with constant holomorphic sectional curvature and constant type. This is equivalent to the following definition. 
DEFINITION 12.3. A generalized complex space form is an almost Hermitian manifold with Riemann curvature tensor $R$ of the following form:

$$
R=\frac{\tau(R)+3 \tau^{*}(R)}{16 n(n+1)}\left(\pi_{1}+\pi_{2}\right)+\frac{\tau(R)-\tau^{*}(R)}{16 n(n-1)}\left(3 \pi_{1}-\pi_{2}\right) .
$$

First we have the following

THEOREM 12.4. An almost Hermitian manifold is Einsteinian and *-Einsteinian if and only if $\left(p_{2}+p_{5}+p_{8}\right)(R)=0$.

Then Theorem 9.4 implies the following characterization:

THEOREM 12.5. An almost Hermitian manifold $M$ is a generalized complex space form if and only if $M$ is Einsteinian, *-Einsteinian and Bochner flat (i.e. $B(R)=0)$.

Of course any Kähler manifold with constant holomorphic sectional curvature is a generalized complex space form. This is also true for any almost Hermitian manifold with constant sectional curvature, as for example $S^{6}$. We do not know of other examples. Now we will prove that these cases are the only possible ones when $2 n \geqslant 6$.

First of all we need

LEMMA 12.6. Let $M$ be an almost Hermitian manifold with Riemannian connection $\nabla$. Then

(i) $\left(\nabla_{X} J\right) J Y=-J\left(\nabla_{X} J\right) Y$,

(ii) $g\left(\left(\nabla_{X} J\right) Y, Z\right)=-g\left(Y,\left(\nabla_{X} J\right) Z\right)$,

(iii) $g\left(\left(\nabla_{X} J\right) Y, Y\right)=0$,

(iv) $g\left(\left(\nabla_{X} J\right) Y, J Y\right)=0$,

for $X, Y, Z \in \mathcal{X}(M)$.

Proof. (i) and (ii) are well known and (iii) and (iv) are easy consequences.

Now we prove the fundamental theorem.

THEOREM 12.7. Let $M$ be a connected almost Hermitian manifold with real dimension $2 n \geqslant 6$ and Riemannian curvature tensor $R$ of the following form:

$$
R=f \pi_{1}+h \pi_{2}
$$

where $f$ and $h$ are $C^{\infty}$ functions on $M$ such that $h$ is not identical zero. Then $M$ is a complex space form (i.e. a Kähler manifold with constant holomorphic sectional curvature).

Proof. Using the expressions of $\pi_{1}$ and $\pi_{2}$ in Theorem 4.1 we obtain at once $\nabla \pi_{1}=0$ and

$$
\begin{aligned}
\left(\nabla_{W} \pi_{2}\right)(X, Y, Z) & =2 g\left(\left(\nabla_{W} J\right) X, Y\right) J Z+g\left(\left(\nabla_{W} J\right) X, Z\right) J Y-g\left(\left(\nabla_{W} J\right) Y, Z\right) J X \\
& +2 g(J X, Y)\left(\nabla_{W} J\right) Z+g(J X, Z)\left(\nabla_{W} J\right) Y-g(J Y, Z)\left(\nabla_{W} J\right) X .
\end{aligned}
$$

Hence we get from (12.1)

$$
\nabla_{W} R=W(f) \pi_{1}+W(h) \pi_{2}+h \nabla_{W} \pi_{2}
$$


and, using the second Bianchi identity, we must have

$$
\underset{W X Y}{\subseteq}\left\{W(f) \pi_{1}(X, Y) Z+W(h) \pi_{2}(X, Y) Z+h\left(\nabla_{W} \pi_{2}\right)(X, Y, Z)\right\}=0 .
$$

Since $\operatorname{dim} M \geqslant 4$ it is possible to choose two unit vectors $X$ and $W$ which define orthogonal holomorphic planes $\{X, J X\}$ and $\{W, J W\}$. Putting then $Z=X$ and $Y=J W$ in (12.2) we derive

$$
\begin{aligned}
2 h\left(\nabla_{X} J\right) X= & -\left\{(J W)(f)+h g\left(\left(\nabla_{X} J\right) X, W\right)\right\} W \\
& +\left\{W(f)-h g\left(\left(\nabla_{X} J\right) X, J W\right)\right\} J W \\
& -\left\{2 X(h)+3 h g\left(\left(\nabla_{W} J\right) W, J X\right)+3 h g\left(\left(\nabla_{J W} J\right) J W, J X\right)\right\} J X .
\end{aligned}
$$

So we obtain

$$
3 h g\left(\left(\nabla_{X} J\right) X, W\right)+(J W)(f)=0
$$

and

$$
2 X(h)+3 h\left\{g\left(\left(\nabla_{W} J\right) W, J X\right)+g\left(\left(\nabla_{J W} J\right) J W, J X\right)\right\}=0 .
$$

Thus $X(h+f)=0$ which implies that $f+h$ is constant on $M$.

Further, using this conclusion, we easily obtain from (12.4) and (12.5) for such pair of vectors:

$$
3 h\left(\nabla_{X} J\right) X=W(f) J W-(J W)(f) W .
$$

Next we put $Z=X$ and $Y=J X$ in (12.2). Then we obtain with Lemma 12.6 and (12.4):

$$
\begin{aligned}
3 h\left(\nabla_{W} J\right) X- & h\left(\nabla_{X} J\right) W \\
& =(J X)(f) W-X(f) J W-\frac{1}{3}(J W)(f) X+\frac{1}{3} W(f) J X .
\end{aligned}
$$

The vectors $\tilde{X}=(X+W) / \sqrt{2}, \tilde{W}=(X-W) / \sqrt{2}$ also determine two orthogonal holomorphic planes. Using these vectors for (12.5) we get

$$
\begin{aligned}
3 h\left\{\left(\nabla_{X} J\right) W\right. & \left.+\left(\nabla_{W} J\right) X\right\} \\
& =-X(f) J W-W(f) J X+(J X)(f) W+(J W)(f) X
\end{aligned}
$$

and so, (12.6) and (12.7) imply

$$
3 h\left(\nabla_{X} J\right) W=(J W)(f) X-W(f) J X .
$$

Finally (12.1) implies that $M$ is Einsteinian. We have explicitly

$$
\rho(R)=\{(2 n-1) f+3 h\} g
$$

and this implies that $(2 n-1) f+3 h$ is constant. Hence, when $n>2$, we have that $f$ and $h$ are both constant. Since $h \neq 0,(12.5)$ and (12.8) imply

$$
\left(\nabla_{X} J\right) X=0, \quad \nabla_{X}(J) W=0
$$

for all unit vector fields which span two orthogonal holomorphic planes. We conclude from this that $\nabla J=0$, i.e. $M$ is a Kähler manifold. So $R \in \mathscr{R}_{1}(M)$ and hence $f=h$, which proves the required result.

REMARKS. (A) When $n=2$, we still have $f+h=$ const. So Theorem 12.6 is still valid if this implies that $f$ and $h$ are both constant functions.

(B) Theorem 12.7 implies that there do not exist almost Hermitian manifolds with $R=h\left(3 \pi_{1}-\pi_{2}\right), h \neq 0$. 
(C) The hypothesis $h \neq 0$ is essential. Indeed, there exist non-Kähler almost Hermitian manifolds which are flat [23], [24]. Moreover, there exist almost Hermitian manifolds with constant sectional curvature which are not Kähler manifolds. $S^{6}$ is a simple example.

(D) S. Sawaki and K. Sekigawa [19] proved by different methods the following special case of Theorem 12.7: Let $M$ be an almost Hermitian manifold with $R \in \mathscr{R}_{1}(M)$ and with constant holomorphic sectional curvature $\mu \neq 0$. Then $M$ is a Kähler manifold.

Manifolds with $R \in \mathscr{R}_{1}(M)$ are there called $F$-spaces.

(E) We state the following problem: Do there exist 4-dimensional almost Hermitian manifolds $M$ with $R=f \pi_{1}+h \pi_{2}$ where $h$ is a nonconstant $C^{\infty}$ function? Of course our proof shows that $M$ must have constant scalar curvature $\tau(R)=12(h+f)$ and $M$ must be Hermitian on $U=\{m \in M \mid h(m) \neq 0\}$ since (12.5) and (12.8) imply $\left(\nabla_{X} J\right) W-\nabla_{J X}(J) J W=0$ for all $X, W \in \mathfrak{X}(M)$.

13. The curvature of the Calabi-Eckmann and Hopf manifolds. In this section we will consider a particular class of almost Hermitian manifolds and discuss some aspects of the decomposition in detail.

We review briefly some of the geometry of the Calabi-Eckmann and Hopf manifolds.

Let $S_{i}^{2 k_{i}+1}\left(r_{i}\right), i=1,2$, be a hypersphere of radius $r_{i}$ in $\mathbf{C}^{k_{1}+1}$ and denote by $N_{i}$, resp. $J_{i}$, a unit normal field, resp. the standard complex structure of $\mathbf{C}^{k_{i}+1}$. An arbitrary tangent field $X$ of

$$
M=S_{1}^{2 k_{1}+1}\left(r_{1}\right) \times S_{2}^{2 k_{2}+1}\left(r_{2}\right)
$$

may be written as

$$
X=X_{1}+a(X) J_{1} N_{1}+X_{2}+b(X) J_{2} N_{2}
$$

where $X_{i}$ is tangent to $S_{i}^{2 k_{i}+1}$. We define an almost Hermitian structure on the product $M$ by

$$
J X=J_{1} X_{1}-b(X) J_{1} N_{1}+J_{2} X_{2}+a(X) J_{2} N_{2}
$$

and the product metric $g$. It is easy to verify that this manifold $(M, J, g)$ is a Hermitian manifold (See also [11].)

For $k_{1}, k_{2} \geqslant 1$ we obtain the Calabi-Eckmann manifolds and for $k_{1}>1, k_{2}=0$ we have a Hopf manifold.

Since we are interested in curvature properties we first compute the Riemann curvature tensor. We denote by $g_{i}$ the metric on $S_{i}^{2 k_{i}+1}$. We obtain

$$
\begin{aligned}
R(X, Y, Z, W)=r^{-2}\left\{g_{1}\left(X_{1}, Z_{1}\right) g_{1}\left(Y_{1}, W_{1}\right)-g_{1}\left(X_{1}, W_{1}\right) g_{1}\left(Y_{1}, Z_{1}\right)\right. \\
+a(X) a(Z) g_{1}\left(Y_{1}, W_{1}\right)+a(Y) a(W) g_{1}\left(X_{1}, Z_{1}\right) \\
\left.-a(X) a(W) g_{1}\left(Y_{1}, Z_{1}\right)-a(Y) a(Z) g_{1}\left(X_{1}, W_{1}\right)\right\} \\
+r_{2}^{-2}\left\{g_{2}\left(X_{2}, Z_{2}\right) g_{2}\left(Y_{2}, W_{2}\right)-g_{2}\left(X_{2}, W_{2}\right) g_{2}\left(Y_{2}, Z_{2}\right)\right. \\
+b(X) b(Z) g_{2}\left(Y_{2}, W_{2}\right)+b(Y) b(W) g_{2}\left(X_{2}, Z_{2}\right) \\
\left.-b(X) b(W) g_{2}\left(Y_{2}, Z_{2}\right)-b(Y) b(Z) g_{2}\left(X_{2}, W_{2}\right)\right\} .
\end{aligned}
$$


From this we deduce

$$
\begin{array}{r}
\rho(R)(X, Y)=\left(2 k_{1} / r_{1}^{2}\right)\left\{g_{1}\left(X_{1}, Y_{1}\right)+a(X) a(Y)\right\} \\
+\left(2 k_{2} / r_{2}^{2}\right)\left\{g_{2}\left(X_{2}, Y_{2}\right)+b(X) b(Y)\right\} \\
\rho^{*}(R)(X, Y)=r_{1}^{-2} g_{1}\left(X_{1}, Y_{1}\right)+r_{2}^{-2} g_{2}\left(X_{2}, Y_{2}\right)
\end{array}
$$

and, further,

$$
\begin{gathered}
\tau(R)=\sum_{i=1}^{2} \frac{2 k_{i}\left(2 k_{i}+1\right)}{r_{i}^{2}} \\
\tau^{*}(R)=\sum_{i=1}^{2} \frac{2 k_{i}}{r_{i}^{2}}
\end{gathered}
$$

First of all we note that $M$ is flat if and only if $k_{1}=k_{2}=0$.

When $k_{1} \geqslant 1, k_{2}=0$ one knows [26], [27] that $M$ is locally conformal to $\mathrm{C}^{2\left(k_{1}+1\right)}$ and hence $M$ is conformally flat and locally conformal to a Kähler manifold.

(13.2) shows that $M$ is Einsteinian if and only if $k_{1} / r_{1}^{2}=k_{2} / r_{2}^{2}$ while (13.3) shows that $\rho^{*}(R)$ is always symmetric but $M$ is never *-Einsteinian in the nonflat case.

Using (13.1)-(13.5) we may compute all the quadratic invariants explicitly. We obtain

$$
\begin{aligned}
& o_{1}(R)=\sum_{i=1}^{2} \frac{4 k_{i}\left(2 k_{i}+1\right)}{r_{i}^{4}}, \quad o_{2}(R)=\sum_{i=1}^{2} \frac{4 k_{i}^{2}\left(2 k_{i}+1\right)}{r_{i}^{4}}, \\
& o_{3}(R)=\left\{\sum_{i=1}^{2} \frac{2 k_{i}\left(2 k_{i}+1\right)}{r_{i}^{2}}\right\}^{2}, \\
& u_{1}(R)=\sum_{i=1}^{2} \frac{4 k_{i}}{r_{i}^{4}}, \quad u_{3}(R)=2 u_{2}(R)=\sum_{i=1}^{2} \frac{4 k_{i}\left(2 k_{i}-1\right)}{r_{i}^{4}}, \\
& u_{4}(R)=u_{7}(R)=\sum_{i=1}^{2} \frac{2 k_{i}}{r_{i}^{4}}, \quad u_{5}(R)=\sum_{i=1}^{2} \frac{4 k_{i}^{2}}{r_{i}^{4}}, \\
& u_{6}(R)=8\left\{k_{1}^{3} / r_{1}^{4}+k_{2}^{3} / r_{2}^{4}+k_{1} k_{2} / r_{1}^{2} r_{2}^{2}\right\}, \\
& u_{8}(R)=\left\{\sum_{i=1}^{2} \frac{2 k_{i}\left(2 k_{i}+1\right)}{r_{i}^{2}}\right\}\left\{\sum_{i=1}^{2} \frac{2 k_{i}}{r_{i}^{2}}\right\},
\end{aligned}
$$

Now it is also possible to compute the square of the length of the tensors $C_{i}(R)$, $i=1, \ldots, 7$, and the projections $p_{j}(R), j=1, \ldots, 10$. By doing this we arrive at the following results.

THEOREM 13.1. We have:

(i) $C_{5}(R)=C_{6}(R)=0$ for all $k_{i}$;

(ii) $M$ is conformally flat if and only if $M$ is flat $\left(k_{1}=k_{2}=0\right)$ or $k_{1}=0, k_{2} \geqslant 1$, resp. $k_{1} \geqslant 1, k_{2}=0$;

(iii) $C_{4}(R)=0$ if and only if $k_{1}=k_{2}=1\left(\operatorname{dim} M=6, k_{i} \neq 0\right)$ or $M$ is conformally flat; 
(iv) $C_{i}(R)=0, i=1,2,3,7$, if and only if $M$ is conformally flat;

(v) $p_{i}(R)=0, i=1,2,4$, if and only if $M$ is flat;

(vi) $p_{5}(R)=0$ if and only if $\operatorname{dim} M=4$ or $M$ is flat;

(vii) $p_{8}(R)=0$ if and only if $M$ is Einsteinian.

14. The decomposition for 4-dimensional spaces. In this section we discuss in more detail the decomposition in the case $n=2$. The special feature of 4-dimensional geometry arises from the particular role of the duality *-operator.

Let $V$ be a 4-dimensional real vector space with positive definite inner product $g$ and $\Lambda^{2}(V)$ the space of skew-symmetric 2-tensors over $V$. Then $R(V)$ is a subspace of the space of symmetric linear operators on $\Lambda^{2}(V)$. We denote by $\langle$, the induced inner product from $g$ on $\Lambda^{2}(V)$. This is given by

$$
\langle x \wedge y, z \wedge w\rangle=\operatorname{det}\left(\begin{array}{ll}
g(x, z) & g(x, w) \\
g(y, z) & g(y, w)
\end{array}\right)
$$

for all $x, y, z, w \in V$. Choose an orientation of $V$ and let $\omega \in \Lambda^{4}(V)$ be the volume element defined by $g$ and the orientation of $V$. Then the Hodge *-operator is the symmetric linear operator defined by $\langle * \xi, \eta\rangle=\langle\xi \wedge \eta, \omega\rangle$ for $\xi, \eta \in \Lambda^{2}(V)$.

First we recall the decomposition of $\Re(V)$ into irreducible factors for the action of the orthogonal group $\Theta(4)$ (see [21]).

THEOREM 14.1. We have

$$
\Re(V)=\mathscr{L}\left(\pi_{1}\right) \oplus \Re_{W}(V) \oplus \Re_{0}(V)
$$

where

$$
\begin{aligned}
\Re_{W}(V) & =\{R \in \mathscr{R}(V) \mid R *=* R\} \cap \mathcal{L}^{\perp}\left(\pi_{1}\right), \\
\Re_{0}(V) & =\{R \in \Re(V) \mid R *=-* R\} .
\end{aligned}
$$

The action of $\Theta(4)$ on $\mathcal{R}(V)$ is irreducible on $\mathcal{L}\left(\pi_{1}\right), \Re_{W}(V)$ and $\Re_{0}(V)$. Further, $\operatorname{dim} \Re_{W}(V)=10$ and $\operatorname{dim} \Re_{0}(V)=9$.

Note that $\mathcal{L}\left(\pi_{1}\right)=\{1\}$ where 1 is the identity transformation on $\Lambda^{2}(V)$.

For the action of $\delta \vartheta(4)$ there is a further orthogonal splitting. We have [21]

THEOREM 14.2. $R(V)=\mathcal{L}\left(\pi_{1}\right) \oplus \Re_{W}^{+}(V) \oplus \Re_{W}^{-}(V) \oplus \Re_{0}(V)$ where

$$
\Re_{W}^{ \pm}(V)=\left\{R \in \Re_{W}(V) \mid R *= \pm R\right\} .
$$

The action of $\mathcal{S}(4)$ is irreducible on each of the factors and $\operatorname{dim} \Re_{W}^{ \pm}(V)=5$.

Let $R \in \mathcal{R}(V)$. Then the projection of $R$ on $\Re_{W}^{+}(V)$ is called the self-dual part of $C(R)$ and the projection on $\Re_{W}^{-}(V)$ the anti-self-dual part of $C(R)$ where $C(R)$ is the Weyl component of $R$. Curvature tensors for which one of these projections vanishes are called half-conformally flat curvature tensors.

Finally we now consider the further decomposition of $\Re(V)$ under the action of थ(2). We give a description of the decomposition with the help of * and the induced map $J: \Lambda^{2}(V) \rightarrow \Lambda^{2}(V)$ defined by $J(x \wedge y)=J x \wedge J y, x, y \in V$, and linearity. Note that on $\Lambda^{2}(V)$ we have $J^{2}=1$. This complete decomposition is also given in [8]. We write it down using our notation. 
THEOREM 14.3. Let $V$ be a 4-dimensional Hermitian vector space. Then we have the following further splitting:

$$
\begin{aligned}
& \Re_{W}^{+}(V)=\mathscr{L}\left(\pi_{1}-\pi_{2}\right) \oplus \mathscr{W}_{7} \oplus \mathscr{W}_{9}, \\
& \Re_{W}^{-}(V)=\mathscr{W}_{3}, \quad \Re_{0}(V)=\mathcal{W}_{2} \oplus \mathcal{W}_{8}
\end{aligned}
$$

where

$$
\begin{aligned}
& \mathcal{L}\left(\pi_{1}-\pi_{2}\right)=\{2 *+3 J-1\}, \\
\mathscr{W}_{2}= & \{R \in \mathcal{R}(V) \mid R *=-* R, R J=R\}, \\
\mathscr{w}_{8}= & \{R \in \mathcal{R}(V) \mid R *=-* R, R J=-J R\}, \\
\mathcal{W}_{3}= & \{R \in \mathcal{R}(V) \mid R *=-R\}, \\
\mathcal{W}_{7}= & \{R \in \mathcal{R}(V) \mid R *=R\} \cap \mathcal{L}^{\perp}\left(\pi_{1}-\pi_{2}\right), \\
\mathcal{W}_{9}= & \{R \in \mathcal{R}(V) \mid R *=R, R J=-J R\} .
\end{aligned}
$$

Further, we have

$\operatorname{dim} \mho_{3}=5, \operatorname{dim} \mho_{7}=2, \operatorname{dim} w_{9}=2, \quad \operatorname{dim} w_{2}=3, \operatorname{dim} \mho_{8}=6$ and the action of $2(2)$ is irreducible on each of the factors.

Using Theorem 9.4 we obtain

THEOREM 14.4. We have $\Re_{B}(V)=\mho_{3} \oplus \mho_{7}$. Further, let $R \in \mathcal{R}(V)$; then the Bochner tensor $B(R)$ is the anti-self-dual part of the Weyl component $C(R)$ if and only if $p_{7}(R)=0$.

Using Theorem 11.4 we derive from Theorem 14.4 and the decomposition:

COROllaRY 14.5. Let $R \in \Re_{1}(V)$ or let $R$ be the Riemann curvature tensor on $a$ Hermitian manifold or a manifold $M \in Q \mathcal{F}_{2}$. Then the Bochner tensor associated with $R$ is the anti-self-dual part of the conformal tensor $C(R)$.

Next we consider the quadratic invariants when $\operatorname{dim} V=2$. We have

THEOREM 14.6. Let $R \in \Re(V)$ and $\operatorname{dim} V=4$. Then there are 8 linear independent quadratic invariants instead of 12 . We have the following relations:

(a) $o_{1}-o_{3}-2 u_{1}+4 u_{2}+u_{3}+2 u_{8}-u_{9}=0$;

(b) $3 o_{1}-6 o_{2}-3 u_{3}-2 u_{4}+6 u_{6}+2 u_{7}=0$;

(c) $2 o_{2}-o_{3}-4 u_{5}+2 u_{6}+u_{8}=0$;

(d) $2 u_{4}-4 u_{5}+2 u_{7}+u_{8}-u_{9}=0$.

Proof. Use Theorems 8.1 and 10.1. (a) expresses that $p_{6}(R)=0$ and (b) expresses that $p_{10}(R)=0$. Finally Theorem 10.1(iii), i.e.

$$
\rho\left(R+L_{3} R\right)-\rho^{*}\left(R+L_{3} R\right)=\frac{1}{2}\left(\tau(R)-\tau^{*}(R)\right) g,
$$

expresses that $p_{5}(R)=0$. Then (c) and (d) follow from this relation by taking the inner product with $\rho(R)$ and $\rho^{*}(R)$.

In the remaining part of this section we will treat some applications of this decomposition for 4-dimensional compact orientable Einstein manifolds. In particular we will consider the Euler-Poincaré characteristic, the Hirzebruch signature 
and the arithmetic genus. The main results are already contained in [8]. (See also [14], [35].) However the author considers only Kähler manifolds.

On a general compact 4-dimensional manifold we have

$$
\chi(M)=\sum_{p=0}^{4}(-1)^{p} \operatorname{dim} h^{p}(M)=\frac{1}{32 \pi^{2}} \int_{M}\left\{\|R\|^{2}-4\|\rho(R)\|^{2}+\tau(R)^{2}\right\} d V
$$

where $d V$ denotes the volume element of $M$ and $h^{p}$ the real vector space of harmonic forms of degree $p$. When $M$ is complex we also have $\chi(M)=\int_{M}{ }^{*} \gamma_{2} d V$ where $\gamma_{2}$ is the second Chern form of $M$. When $M$ is an Einstein space (14.1) becomes

$$
\chi(M)=\frac{1}{32 \pi^{2}} \int_{M}\|R\|^{2} d V
$$

and, since $p_{2}(R)=p_{8}(R)=0$, we have

$$
\chi(M)=\frac{1}{32 \pi^{2}} \int_{M}\left\{\frac{\tau(R)^{2}}{6}+\left\|W^{+}(R)\right\|^{2}+\left\|W^{-}(R)\right\|^{2}\right\} d V
$$

where $W^{+}(R)$, resp. $W^{-}(R)$, denotes the self-dual, resp. anti-self-dual, part of $C(R)$.

Now let $M$ be orientable and let $h^{2}(M)=h_{+}(M) \oplus h_{-}(M)$, where $h_{ \pm}(M)$ is the eigenspace of * with eigenvalues \pm 1 . Then the Hirzebruch signature $\tau(M)$ is given by

$$
\tau(M)=\operatorname{dim} h_{+}(M)-\operatorname{dim} h_{-}(M)=\frac{1}{48 \pi^{2}} \int_{M}\langle R, R *\rangle d V .
$$

When $M$ is complex we also have

$$
\tau(M)=\frac{1}{3} \int_{M} *\left(\gamma_{1}^{2}-2 \gamma_{2}\right) d V=\frac{1}{3} \int_{M} * p_{1} d V=-\frac{1}{48 \pi^{2}} \int_{M}\left\{\|R\|^{2}-2\|\rho\|^{2}\right\} d V
$$

where $p_{1}$ is the first Pontryagin form and $\gamma_{1}^{2}$ the square of the first Chern form. Using the decomposition, (14.3) becomes

$$
\tau(M)=\frac{1}{48 \pi^{2}} \int_{M}\left\{\left\|W^{+}(R)\right\|^{2}-\left\|W^{-}(R)\right\|^{2}\right\} d V .
$$

From (14.2) and (14.4) we have

THEOREM 14.7 [8]. Let $M$ be a compact oriented 4-dimensional Einstein manifold. Then:

$$
|\tau(M)|<\frac{2}{3} \chi(M)-\left(\tau(R)^{2} / 288 \pi^{2}\right) \operatorname{vol}(M)
$$

(ii) $\quad \tau(M)=-\frac{2}{3} \chi(M)+\left(\tau(R)^{2} / 288 \pi^{2}\right) \operatorname{vol}(M)$ if and only if $W^{-}(R)=0$;

(iii) $\quad \tau(M)=\frac{2}{3} \chi(M)-\left(\tau(R)^{2} / 288 \pi^{2}\right) \operatorname{vol}(M)$ if and only if $W^{+}(R)=0$.

We refer to [8] for results concerning the possibilities of $\chi(M)$ and $\tau(M)$ on Ricci flat manifolds and for other useful inequalities. 
From now on we consider only 4-dimensional almost Hermitian Einstein manifolds with the orientation defined by the almost complex structure. Then we have

$$
W^{+}(R)=C_{1}(R)+p_{7}(R)+p_{9}(R), \quad W^{-}(R)=p_{3}(R),
$$

and

$$
\left\|W^{+}(R)\right\|^{2}=\left(\tau(R)-3 \tau^{*}(R)\right)^{2} / 24+\left\|p_{7}(R)\right\|^{2}+\left\|p_{9}(R)\right\|^{2} .
$$

Note that a 4-dimensional almost Hermitian Einstein manifold belongs to the class $Q \mathcal{F G}_{2}$ if and only if $p_{7}(R)=p_{9}(R)=0$. Hence we have

THEOREM 14.8. Let $M$ be a 4-dimensional almost Hermitian Einstein space. Then $W^{+}(R)=0$ if and only if $M \in \mathbb{Q} \mathcal{F}_{2}$ and $\tau(R)=3 \tau^{*}(R)$. If $M \in \mathbb{Q} \mathcal{F}_{1}$ then $W^{+}(R)=0$ if and only if $M$ is Ricci flat.

Further, we have, just as in the Kählerian case,

THEOREM 14.9. Let $M \in \mathbb{Q} \mathcal{H}_{1}$. Then

$$
\begin{gathered}
\chi(M)=\frac{1}{32 \pi^{2}} \int_{M}\left\{\frac{\tau(R)^{2}}{3}+\left\|W^{-}(R)\right\|^{2}\right\} d V ; \\
\chi(M)=\frac{1}{48 \pi^{2}} \int_{M}\left\{\frac{\tau(R)^{2}}{6}-\left\|W^{-}(R)\right\|^{2}\right\} d V ; \\
\chi(M)+\frac{3}{2} \tau(M)=\frac{\tau(R)^{2}}{64 \pi^{2}} \operatorname{vol}(M), \quad \chi(M)-3 \tau(M)=\frac{3}{32 \pi^{2}} \int_{M}\left\|W^{-}(R)\right\|^{2} d V ; \\
-2 \chi(M) \leqslant 3 \tau(M) \leqslant \chi(M) .
\end{gathered}
$$

When $M \in \mathbb{Q} \mathcal{F}_{1}, W^{-}(R)=0$ if and only if $M$ has constant holomorphic sectional curvature. Hence, in the nonflat case, $\mathbf{C} P^{2}(\mu)$ is the standard example. It would be interesting to know if there are other examples. When we take Einstein manifolds of the class $Q \mathcal{F G}_{2}$, then $W^{-}(R)=0$ if and only if $M$ is a generalized complex space form. But for dimension 4 this is just the case that remained open in $\S 12$. We do not know of any example which is compact and not Kählerian.

Examples of compact manifolds with $W^{+}(R)=0$ are given by those which are locally isometric to a $K_{3}$-surface. We do not know if there are examples which are not Kählerian.

In the noncompact case we gave in [31] an example of a Hermitian manifold of the class $Q \mathcal{F H}_{2}$ with $W^{+}(R)=W^{-}(R)=0$ and which is Einsteinian.

We finish this section by considering the arithmetic genus $\alpha(M)$ for an oriented compact 4-dimensional manifold. This is defined by

$$
\alpha(M)=\frac{1}{4}(\chi(M)+\tau(M)) .
$$

When $M$ is a complex manifold with the orientation defined by the complex structure, then

$$
\begin{aligned}
\alpha(M) & =\frac{1}{12}\left(\chi(M)+c_{1}^{2}(M)\right)=\frac{1}{12} \int_{M} *\left(\gamma_{1}^{2}+\gamma_{2}\right) d V \\
& =\frac{1}{384 \pi^{2}} \int_{M}\left\{\|R\|^{2}-8\|\rho(R)\|^{2}+3 \tau(R)^{2}\right\} d V
\end{aligned}
$$


where $c_{1}^{2}(M)$ denotes the square of the first Chern number. In that case $\alpha(M)$ coincides with the usual definition of the arithmetic genus. Further, we have in that case

$$
\alpha(M)=\sum_{p=0}^{2}(-1)^{p} \operatorname{dim} h^{p, 0}
$$

where $h^{p, 0}$ is the space of harmonic forms of bidegree $(p, 0)$. Using (14.2) and (14.4) we obtain for an Einstein manifold:

$$
\alpha(M)=\frac{1}{384 \pi^{2}} \int_{M}\left\{\frac{\tau(R)^{2}}{2}+5\left\|W^{+}(R)\right\|^{2}+\left\|W^{-}(R)\right\|^{2}\right\} d V,
$$

and hence we obtain with (14.2) and (14.4)

THEOREM 14.10. Let $M$ be a compact 4-dimensional almost Hermitian Einstein manifold. Then we have

$$
\begin{gathered}
\frac{1}{12} \chi(M)+\frac{\tau(R)^{2}}{1152 \pi^{2}} \operatorname{vol}(M) \leqslant \alpha(M) \leqslant \frac{5}{2} \chi(M)-\frac{\tau(R)^{2}}{1152 \pi^{2}} \operatorname{vol}(M), \\
-\alpha(M)+\frac{\tau(R)^{2}}{768 \pi^{2}} \operatorname{vol}(M) \leqslant \frac{1}{8} \tau(M) \leqslant \frac{1}{5} \alpha(M)-\frac{\tau(R)^{2}}{3840 \pi^{2}} \operatorname{vol}(M),
\end{gathered}
$$

where the left equality sign is valid if and only if $W^{+}(R)=0$ and the right equality sign if and only if $W^{-}(R)=0$.

THEOREM 14.11. Let $M$ be a compact 4-dimensional almost Hermitian Einstein manifold of the class $\mathbb{Q S F}_{2}$. Then

$$
\begin{aligned}
\alpha(M)-\frac{1}{12} \chi(M) & =\frac{1}{1152 \pi^{2}} \int_{M}\left\{\tau(R)^{2}+\frac{\left(\tau(R)-3 \tau^{*}(R)\right)^{2}}{2}\right\} d V . \\
\alpha(M)+\frac{1}{8} \tau(M) & =\frac{1}{768 \pi^{2}} \int_{M}\left\{\tau(R)^{2}+\frac{\left(\tau(R)-3 \tau^{*}(R)\right)^{2}}{2}\right\} d V . \\
\chi(M)+\frac{3}{2} \tau(M) & =\frac{1}{192 \pi^{2}} \int_{M}\left\{\tau(R)^{2}+\frac{\left(\tau(R)-3 \tau^{*}(R)\right)^{2}}{2}\right\} d V .
\end{aligned}
$$

We refer also to [4] for interesting inequalities between $\chi(M), \tau(M)$ and $\alpha(M)$ in the Kähler case.

15. Submanifolds and the Bochner tensor. In this section we first prove a theorem which relates the Bochner tensor of an almost Hermitian manifold with the Weyl tensor of a special type of submanifold.

Let $(M, g, J)$ be an almost Hermitian manifold and $N$ a submanifold of $M . N$ is said to be a holomorphic submanifold of $M$ if $J T_{m}(N)=T_{m}(N)$ for all $m \in N$ and $N$ is a totally real submanifold if for all $m \in N$ we have $J T_{m}(N) \subset T_{m}^{\perp}(N)$, where $T_{m}^{\perp}(N)$ denotes the normal space of $T_{m} N$ in $T_{m} M$. Further, denote by $\tilde{\nabla}$ the induced Riemannian connection on $N$. Then

$$
\nabla_{X} Y=\tilde{\nabla}_{X} Y+\sigma(X, Y)
$$

for all $X, Y \in \mathscr{X}(N)$. $\sigma$ is the so-called second fundamental form. 
$N$ is called a totally quasi-umbilical submanifold if

$$
g\left(\sigma(X, Y), E_{i}\right)=\lambda_{i} g(X, Y)+\mu_{i} \eta_{i}(X) \eta_{i}(Y)
$$

for each $E_{i}$ of an orthonormal basis $\left\{E_{i}\right\}$ of $T_{m}^{\perp} N$, the $\eta_{i}$ being 1-forms. This means that the second fundamental form in the direction of the normal vector $E_{i}$ has only two eigenvalues: one with multiplicity $\operatorname{dim} N-1$ and one with multiplicity $1 . N$ is totally geodesic when $\lambda_{i}=\mu_{i}=0$ and totally umbilical when $\mu_{i}=0$ for all $i$.

Finally the Gauss equation is given by

$$
\begin{aligned}
R(X, Y, Z, W)= & \tilde{R}(X, Y, Z, W) \\
& +g(\sigma(X, Z), \sigma(Y, W))-g(\sigma(X, W), \sigma(Y, Z))
\end{aligned}
$$

where $\tilde{R}$ is the Riemann curvature tensor of $N$ and $X, Y, Z, W \in \mathcal{X}(N)$.

We will need the following:

LEMMA 15.1 [20]. Let $N^{m}$ be a Riemannian manifold with $\operatorname{dim} N=m>3$ and Riemann curvature tensor $\tilde{R}$. Then $N^{m}$ is conformally flat if and only if $\tilde{R}(X, Y, Z, W)=0$ for all quadruples of orthogonal $X, Y, Z, W \in \mathcal{X}\left(N^{m}\right)$.

Then we have

THEOREM 15.2. Let $M^{2 n}$ be an almost Hermitian manifold with real dimension $2 n \geqslant 8$. If $M$ is Bochner flat, then each totally quasi-umbilical (in particular, totally geodesic or totally umbilical) totally real submanifold $N$ of dimension $>3$ is conformally flat.

Proof. Since $N$ is totally quasi-umbilical we obtain from (15.1) and (15.2) that $R(X, Y, Z, W)=\tilde{R}(X, Y, Z, W)$ for any quadruple of orthogonal $X, Y, Z, W \in$ $\mathcal{X}(N)$. Next, since $B(R)=0$ we obtain from Theorem 9.4 that $\tilde{R}(X, Y, Z, W)=0$. This together with Lemma 15.1, gives the required result.

This theorem generalizes a result for manifolds of the class $\mathbb{Q} \mathcal{H}_{1}$ [32].

Next we consider the case of a holomorphic submanifold $N$ of $M$ and put $V=T_{m} M, \tilde{V}=T_{m} N$ for all $m \in N$. Then we may write (15.2) as follows:

$$
R_{\tilde{V}}=\tilde{R}+H
$$

where $R_{\tilde{V}}$ denotes the restriction of $R$ to $\tilde{V} . R_{\tilde{V}}, \tilde{R}$ and $H$ are curvature tensors belonging to $\Re(\tilde{V})$. Now we prove

THEOREM 15.3. Let $M$ be an almost Hermitian manifold and $N$ a holomorphic submanifold of $M$. Further, let $M$ be Bochner flat. Then $N$ is Bochner flat if and only if the Bochner tensor associated with the curvature tensor $H$ vanishes.

Proof. The Riemann curvature tensor $R$ of $M$ can be written as $R=\sum_{i=1}^{10} p_{i}(R)$ where

$$
\begin{gathered}
p_{1}(R)=\lambda\left(\pi_{1}+\pi_{2}\right), \quad p_{2}(R)=(\varphi+\psi)\left(S_{1}\right), \quad p_{4}(R)=\mu\left(3 \pi_{1}-\pi_{2}\right), \\
p_{5}(R)=(3 \varphi-\psi)\left(S_{2}\right), \quad p_{8}(R)=\varphi\left(S_{3}\right), \quad p_{9}(R)=\psi\left(S_{4}\right)
\end{gathered}
$$


for some $S_{1} \in \mathcal{V}_{1}, S_{2} \in \mathcal{V}_{1}, S_{3} \in \mathcal{V}_{2}$ and $S_{4} \in \mathcal{V}_{3}$. Further we denote by $\sim$ the corresponding notions on $N$ and consider the restrictions $p_{i}(R)_{\tilde{V}}$ of $p_{i}(R)$ to $\tilde{V}$. We have

$$
\tilde{\pi}_{1}=\pi_{1 \tilde{V}}, \quad \tilde{\pi}_{2}=\pi_{2 \tilde{V}}, \quad \tilde{\varphi}\left(S_{\tilde{V}}\right)=\varphi(S)_{\tilde{V}}, \quad \tilde{\psi}\left(S_{\tilde{V}}\right)=\psi(S)_{\tilde{V}}
$$

Also we have that $R_{\tilde{V}} \in \mathscr{R}_{i}(\tilde{V})$ when $R \in \Re_{i}(V)$.

Hence we obtain at each point $m \in N$ :

$$
\begin{aligned}
R_{\tilde{V}}= & \lambda(m)\left(\tilde{\pi}_{1}+\tilde{\pi}_{2}\right)+(\tilde{\varphi}+\tilde{\psi})\left(S_{1 \tilde{V}}\right)+p_{3}(R)_{\tilde{V}}+\mu(m)\left(3 \tilde{\pi}_{1}-\tilde{\pi}_{2}\right) \\
& +(3 \tilde{\varphi}-\tilde{\psi})\left(S_{2 \tilde{V}}\right)+p_{6}(R)_{\tilde{V}}+p_{7}(R)_{\tilde{V}}+\tilde{\varphi}\left(S_{3 \tilde{V}}\right)+\tilde{\psi}\left(S_{4 \tilde{V}}\right)+p_{10}(R)_{\tilde{V}},
\end{aligned}
$$

and so it follows from the arguments made above that:

$$
\begin{gathered}
\tilde{\pi}_{1}+\tilde{\pi}_{2} \in \mathcal{W}_{1}(\tilde{V}), \quad(\tilde{\varphi}+\tilde{\psi})\left(S_{1}\right) \in \mathcal{W}_{1}(\tilde{V}) \oplus \mathcal{W}_{2}(\tilde{V}), \\
p_{3}(R)_{\tilde{V}} \in \mathcal{W}_{1}(\tilde{V}) \oplus \mathcal{W}_{2}(\tilde{V}) \oplus \mathcal{W}_{3}(\tilde{V}), \quad 3 \tilde{\pi}_{1}-\tilde{\pi}_{2} \in \mathcal{W}_{4}(\tilde{V}), \\
(3 \tilde{\varphi}-\tilde{\psi})\left(S_{2 \tilde{V}}\right) \in \mathcal{W}_{4}(\tilde{V}) \oplus \mathcal{W}_{5}(\tilde{V}), \quad p_{6}(R)_{\tilde{V}} \in \mathcal{W}_{4}(\tilde{V}) \oplus \mathcal{W}_{5}(\tilde{V}) \oplus \mathcal{W}_{6}(\tilde{V}), \\
p_{7}(R)_{\tilde{V}} \in \mathcal{W}_{7}(\tilde{V}), \quad \tilde{\varphi}\left(S_{3} \tilde{V}\right) \in \mathcal{W}_{8}(\tilde{V}), \quad \tilde{\psi}\left(S_{4}\right) \in \mathcal{W}_{9}(\tilde{V}), \\
p_{10}(R)_{\tilde{V}} \in \mathcal{W}_{8}(\tilde{V}) \oplus \mathcal{W}_{9}(\tilde{V}) \oplus \mathcal{W}_{10}(\tilde{V}) .
\end{gathered}
$$

Since $M$ is Bochner flat we have from Theorem 9.4:

$$
p_{3}(R)_{\tilde{V}}=p_{6}(R)_{\tilde{V}}=p_{7}(R)_{\tilde{V}}=p_{10}(R)_{\tilde{V}}=0 .
$$

Hence (15.3) and the results above imply that $B(\tilde{R})=0$ if and only if $B(H)=0$, which proves the required result.

COROllary 15.4. Let $M$ be a Bochner flat almost Hermitian manifold and $H a$ holomorphic submanifold of $M$ which satisfies one of the following conditions:

(i) $N$ is totally geodesic;

(ii) $N$ is totally umbilical;

(iii) $N$ is totally quasi-umbilical.

Then $N$ is Bochner flat.

Proof. Using (15.1) it is easy to check that $B(H)=0$ when $N$ is totally quasi-umbilical. Further (i) and (ii) are special cases of (iii).

Note that for Kähler manifolds $M$, cases (ii) and (iii) reduce to (i).

\section{REFERENCES}

1. M. F. Atiyah, N. J. Hitchin and I. M. Singer, Self-duality in four-dimensional Riemannian geometry, Proc. Roy. Soc. London Ser. A 362 (1978), 425-461.

2. M. Berger, P. Gauduchon and E. Mazet, Le spectre d'une variété riemannienne, Lecture Notes in Math., vol. 194, Springer-Verlag, Berlin and New York, 1971.

3. J. Dieudonné and J. Carell, Invariant theory, old and new, Adv. in Math. 4 (1970), 1-80.

4. H. Donnelly, Topology and Einstein Kähler metrics, J. Differential Geom. 11 (1976), 259-264.

5. __ Invariance theory of Hermitian manifolds, Proc. Amer. Math. Soc. 58 (1976), 229-233.

6. P. Gilkey, Spectral geometry and the Kaehler condition for complex manifolds, Invent. Math. 26 (1974), 231-258.

7. A. Gray, Some examples of almost Hermitian manifolds, Illinois J. Math. 10 (1966), 353-566.

8. , Invariants of curvature operators of four-dimensional Riemannian manifolds, Canadian Math. Congress Proc. of the 13th Biennial Seminar, vol. 2, 1971, pp. 42-65.

9. , The structure of nearly Kähler manifolds, Math. Ann. 223 (1976), 233-248. 
10. , Curvature identities for Hermitian and almost Hermitian manifolds, Tôhoku Math. J. 28 (1976), 601-612.

11. A. Gray and L. M. Hervella, The sixteen classes of almost Hermitian manifolds, Ann. Mat. Pura Appl. 123 (1980), 35-58.

12. A. Gray and L. Vanhecke, Almost Hermitian manifolds with constant holomorphic sectional curvature, Czechoslovak Math. J. 104 (1979), 170-179.

13. __ Decomposition of the space of covariant derivatives of curvatures operators (to appear).

14. N. Hitchin, Compact four-dimensional Einstein manifolds, J. Differential Geom. 9 (1974), 435-441.

15. N. Iwahori, Some remarks on tensor invariants of $\Theta(n), ~ U(n), \delta p(n)$, J. Math. Soc. Japan 10 (1958), 145-160.

16. D. L. Johnson, $A$ normal form for curvature, Ph. D. Thesis, M.I.T., 1977 (to appear under the title Critical behaviour of sectional curvature and curvature normal forms for Kähler manifolds).

17. H. Mori, On the decomposition of generalized K-curvature tensor fields, Tôhoku Math. J. 25 (1973), 225-235.

18. K. Nomizu, On the decomposition of generalized curvature tensor fields, Differential geometry (in honor of K. Yano), Kinokuniya, Tokyo, 1972, pp. 335-345.

19. S. Sawaki and K. Sekigawa, Almost Hermitian manifolds with constant holomorphic sectional curvature, J. Differential Geom. 9 (1974), 123-134.

20. J. A. Schouten, Ricci-calculus, Springer-Verlag, Berlin and New York, 1954.

21. I. M. Singer and J. A. Thorpe, The curvature of 4-dimensional Einstein spaces, Global Analysis (papers in honor of K. Kodaira), Univ. of Tokyo Press, Tokyo, 1969, pp. 355-365.

22. M. Sitaramayya, Curvature tensors in Kähler manifolds, Trans. Amer. Math. Soc. 183 (1973), 341-351.

23. F. Tricerri and L. Vanhecke, An example of a 6-dimensional flat almost Hermitian manifold, Colloq. Math. 50 (1978), 111-117.

24. $249-254$.

25. , Flat almost Hermitian manifolds which are not Kähler manifolds, Tensor 31 (1977), spectrum theory, Simon Stevin 53 (1979), 163-173.

26. I. Vaisman, On locally conformal almost Kähler manifolds, Israel J. Math. 24 (1976), 338-351.

27. __ Some curvature properties of locally conformal Kähler manifolds, Trans. Amer. Math. Soc. 259 (1980), 439-447.

28. L. Vanhecke, The Bochner curvature tensor on almost Hermitian manifolds, Rend. Sem. Mat. Univ. Politec. Torino 34 (1975-76), 21-38.

29. On the decomposition of curvature tensor fields on almost Hermitian manifolds, Proc. Conf.

Differential Geometry, Michigan State Univ., East Lansing, Mich., 1976, pp. 16-33.

30. L. Vanhecke and F. Bouten, Constant type for almost Hermitian manifolds, Bull. Math. Soc. Sci.

Math. R. S. Roumanie 20 (1976), 415-422.

31. L. Vanhecke, Almost Hermitian manifolds, Notes, Dept. of Math., Michigan State Univ., East Lansing, Mich., 1976, pp. 1-29.

32. (The Bochner curvature tensor on almost Hermitian manifolds, Geom. Dedicata 6 (1977), $389-397$.

33. L. Vanhecke and D. Janssens, The Bochner curvature tensor on almost Hermitian manifolds, Hokkaido Math. J. 7 (1978), 252-258.

34. H. Weyl, Classical groups, their invariants and representations, Princeton Univ. Press, Princeton, N. J., 1946.

35. Y. L. Xin, Remarks on characteristic classes of four-dimensional Einstein manifolds, J. Math. Phys. 21 (1980), 343-346.

Istituto Matematico, Politecnico di Torino, Corso Duza degli Abruzzi 24, 10129 Torino, ITALIA

Departement Wiskunde, Katholieke Universiteit leuven, Celestiunenlaan 200B, B-3030 LeUVEN BeLGIUM 